\title{
T-mixer operating with water at different temperatures: simulation and stability analysis
}

\author{
L. Siconolfi ${ }^{1}$, S. Camarri ${ }^{1}$ and M.V. Salvetti ${ }^{2}$ \\ ${ }^{1}$ DICI, Università di Pisa, Via Girolamo Caruso, 56122 Pisa, Italy
}

\begin{abstract}
In this paper we investigate the transition from the vortex to the engulfment regime in a Tmixer when the two entering flows have different viscosity. In particular we consider as working fluid water entering the two inlet channels of the mixer at two different temperatures. Contrary to the isothermal case, at low Reynolds numbers the vortex regime shows only a single reflectional symmetry, due to the non-homogeneous distribution of the viscosity. Increasing the Reynolds number, a symmetry-breaking bifurcation drives the system to a new steady flow configuration, usually called engulfment regime, similar to what it is possible to observe in an isothermal case. This flow regime is associated with an increase of the mixing between the two inlet streams. It is shown by Direct Numerical Simulation (DNS) and by stability analysis that the engulfment regime is promoted by the temperature difference.

Starting from the DNS simulations, the resulting flow fields are analysed in detail considering different temperature jumps between the two inlet boundaries. Furthermore, dedicated linear stability analyses are carried out to investigate the instability mechanism associated with the occurrence of the engulfment regime. In particular, similarly to the case without temperature differences, the onset of engulfment is driven by the momentum equation and the temperature field does not lead to any additional instability mechanism. However, the existence of a temperature field leads to quantitative changes of the stability characteristics and of the resulting flow fields via a variation of the viscosity coefficient.
\end{abstract}




\section{INTRODUCTION}

Promoting mixing in a small volume is a pursued goal in many fluid dynamics applications. Recently large attention has been paid to the process intensification in micro-devices for reactions of interest in the field of fine chemistry and for pharmaceutical processes. Small reaction volumes, well controlled residence times and large heat transfer rates (due to the large surface to volume ratio) enable reactions to be carried out under less dilute and extremely controlled conditions, resulting in higher yields than those that can be obtained with conventional batch reactors. Indeed, it has been shown that micro-reactors may be particularly advantageous for several reactions of pharmaceutical [? ] and fine chemistry processes [? ? ], as well as for green chemistry [? ], due to their potential of reducing waste of material and energy. For instance, Roberge et al [? ] showed how about 50\% of the chemical reactions involved in pharmaceutical process could benefit of micro-reaction technologies. Finally, scaling to large productions is trivial when using micro-devices as it can be obtained by increasing their number while working in parallel. Logically in case of chemical reactions the mixing between the reactants is fundamental (see e.g. [? ]), so that the Reynolds number should be high enough to promote convective contribution to the mixing while the flow must remain laminar for a fine control of the residence time. In this respect the onset of the engulfment regime is important since it leads to substantial convective mixing while, before its onset, the two streams entering the mixer remain segregated and mix only due to weak diffusive effects. In particular, we focus on T-shaped micro-mixers which are often employed due to the simplicity of their geometry. For this reason, mixing capabilities of T-mixers have been largely investigated in the literature, in particular in connection with the different flow regimes that can be occured in T-mixers as the Reynolds number is increased. These flow regimes have been largely investigated experimentally (see, for example, [? ? ? ]) and by numerical simulations (see [? ? ? ? ? ? ? ? ]), considering rectangular or square cross sections of the inlet and outlet channels. Despite the quantitative differences related to the geometry (see for instance [? ? ]), it is shown that, at very low values of Re, the inlet streams remain completely segregated, while increasing the Reynolds number the so-called "vortex regime" occurs, i.e. a double pair of counter-rotating vortical structures are originated at the confluence region. Since the flow in the vortical regime preserves the geometrical reflectional symmetries, the two inlet streams 
remain segregated. As $R e$ exceeds a critical value $\left(R e_{c r}\right)$, the flow undergoes a pitchfork bifurcation which breaks the reflectional symmetries and leads to a new steady state, usually called "engulfment regime". The velocity field in the engulfment regime has a central point symmetry with respect to the axis of the outflow channel, and it is characterised by two co-rotating vortices in the outflow channel. This flow regime is associated with a significant enhancement of mixing in comparison with the vortex regime. Because of the increase in mixing, many efforts have been made to predict the critical Re at which the transition to the engulfment regime occurs and to understand its dependence on geometric parameters ([? ? ? ]). Stability analysis has been used in [10] to investigate in detail the physical mechanisms causing the onset of the engulfment regime. By further increasing the value of Re the flow becomes first periodic in time through an Hopf bifurcation (see [? ? ]), and eventually chaotic (see [? ? ? ? ? ]).

In applications involving mixing, T-mixers are always used for mixing two flows with different physical properties, which can be the case of two flows of the same fluid at different conditions or two flows of different fluids. However, all the studies mentioned above on the characterisation of the flow in T-mixers are focused on the case of one homogeneous working fluid, while different operative conditions can have important effects on the identified flow regimes, on the physical mechanisms causing their onset and on the related mixing properties. However, in the practical use of T-mixers, e.g. in pharmaceutical and fine chemistry industry, the two inlet streams have often different physical properties (e.g. two different entering fluids or the same fluid in different conditions).

A few studies in the literature explore this aspect. For instance, [? ] considers the case in which water and ethanol enter a T-mixer, showing that the onset of engulfment is delayed in terms of $R e_{c r}$ from $R e_{c r} \simeq 140$ when water-water is used to $R e_{c r} \simeq 230$ for water-ethanol. Indeed, although water and ethanol have similar viscosity and density, their mixture has a viscosity which is about 3 times larger than that of the single components. The case of a mixtures with strongly varying viscosity has been investigated numerically in [? ], showing that $R e_{c r}$ increases with the fluidity of the mixture. Lastly, in [? ] a systematic study is proposed about the effect of several mixing laws for viscosity in binary mixtures on the flow regimes in a T-mixer. Effect of viscosity ratios between the two entering fluids on the flow regimes in T-mixers is also studied numerically in [? ]. In [? ] the same authors investigate the effect of variable density and viscosity, including the case of water at different 
temperatures similarly to what considered here.

In this paper we focus on the engulfment regime and we investigate using Direct Numerical Simulations (DNS) and dedicated stability analyses, how the onset of the engulfment regime is affected by significant variations of viscosity between the two flows entering the mixer. In order to deal with a realistic case, but still with the aim to deduce general conclusions, we consider water entering the mixer at different temperatures, as viscosity of water is strongly affected by temperature. The objective of the investigation is to explore the role played by the temperature field and by the associated viscosity field on the physical mechanisms which are responsible for the onset of the engulfment steady regime. Moreover, differences in the velocity and vorticity fields inside the mixer and in the associated mixing properties are also investigated in detail by DNS and compared with the case of one homogeneous working fluid. We consider here the same geometrical configuration already investigated in [? ], where the onset of engulfment regime was studied by the synergic use of DNS and stability analysis, as in the present work.

Accurate numerical simulations are carried out using the spectral element method implemented in the code Nek5000, which is used for the 3D simulations of the different cases here considered. Stability analyses have been carried out using ad-hoc 3D tools developed by FreeFem ++ using Taylor-Hood finite elements. We underline that this kind of 3D stability analysis is at the state-of-the-art from a computational viewpoint. Examples of previous stability and sensitivity analysis of incompressible flows in mixers are documented in [? ? ? ? ] and have been carried out considering only one homogeneous working fluid. The theoretical tools used here are similar, with the difference that since we consider water at different temperatures, the equations governing the flow include the energy equation, which is two-way coupled with the momentum equation as the viscosity and thermal diffusivity are functions of the temperature. Examples of stability and sensitivity analysis including the energy equation can be found in the literature (see for instance [? ? ? ] where compressible flows in subsonic regime are considered). In [? ] the stability analysis of a cold incompressible jet impinging on a confined flat plate is carried out considering the temperature as a passive scalar, i.e. with the energy equation which is coupled to the momentum equations only in one way.

This paper clearly demonstrates that stability analysis is able to identify the bifurcation which is responsible for the onset of the engulfment regime, as for the case with no tem- 
perature difference between the two inlet fluids. Moreover, stability analysis is used here so as to highlight the role of the temperature and viscosity variations in physical mechanisms leading to the engulfment instability.

\section{FLOW CONFIGURATION, FLUID PROPERTIES AND GOVERNING EQUA- TIONS}

We consider the T-mixer sketched in Figure ??a. The mixer is made by two symmetric inflow and one outflow channels, measuring $l_{i}$ and $l_{o}$ in length, respectively. The crosssections of the channels are rectangular, with $w / h=0.75$ for the inflow channels and $w_{0} / h=1.5$ for the outflow one, the width of the outflow channel being twice that of the inflow ones, i.e $w_{0}=2 w$. The same geometry has been investigated in [? ? ], focusing on the flow regimes that occur when one incompressible and Newtonian working fluid is employed.

The velocity profile of the flow is assumed to have a fully developed laminar velocity profile when entering the mixer (see for instance [? ]). Moreover, we assume that the working fluid, specifically water, enters the mixer from the two inlet channels at two different temperatures. One of the two temperatures is fixed to $T_{1}=10^{\circ} \mathrm{C}$, while the other one is varied in the range $10^{\circ} \mathrm{C} \leq T_{2} \leq 50^{\circ} \mathrm{C}$. In this range of temperatures water is modeled as a Newtonian fluid with a viscosity $\mu$ that depends on temperature $T$. The behaviour of $\mu(T)$ as $T$ is varied, taken from [? ? ], is sketched in figure ??a in red dots. Here we approximate the law $\mu(T)$ as follows (details are provided in the two references cited above):

$$
\mu(T)=\mu_{1}\left[1+a_{1}\left(T-T_{1}\right)+a_{2}\left(T-T_{1}\right)^{2}\right]^{-1}
$$

where for water $\mu_{1}=\mu\left(T_{1}\right) \simeq 1.307 \cdot 10^{-3} \mathrm{Ns} / \mathrm{m}^{2}$ and the coefficients, $a_{1} \simeq 2.935 \cdot 10^{-2} \mathrm{~K}^{-1}$,

$a_{2} \simeq 1.302 \cdot 10^{-3} \mathrm{~K}^{-2}$, are tuned so as to replicate the experimental behaviour of $\mu(T)$ in the considered range of temperatures, as shown in figure ??a with a blue line. Relative variations of density, which are less than $1.18 \%$ for the maximum temperature difference $T_{2}-T_{1}=40^{\circ} \mathrm{C}$ considered here, are neglected in the flow model, assuming a constant density $\rho$. Also, buoyancy force deriving from density variations is not taken into account, thus excluding the action of gravity forces on the fluid. This assumption has been verified to have negligible effects on the results by means of dedicated numerical simulations, not 

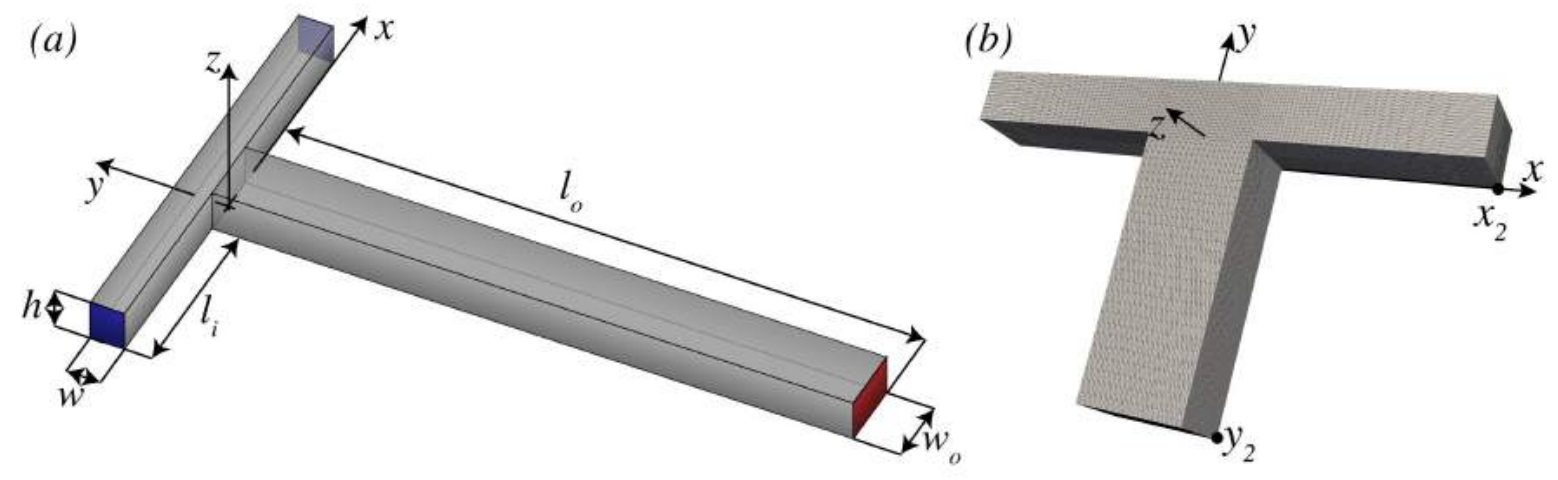

Figure 1. Flow configuration. (a) Computational domain used for DNSs; (b) Computational domain used for stability analysis.

documented here for the sake of brevity.

Finally, the specific heat at constant pressure $c_{p}$ for water is also considered constant with temperature, its relative variations being approximately equal to $0.17 \%$ for the maximum temperature difference considered. Thus, water is modelled here as a Newtonian incompressible fluid with viscosity $\mu$ and thermal conductivity $k$ which vary with temperature. The behaviour of $k(T)$ is given indirectly by specifying the Prandtl number $\operatorname{Pr}=\left(c_{p} \mu\right) / k$ as a function of temperature, this term entering directly in the normalised flow equations shown in the following. The behaviour of $\operatorname{Pr}$ with the temperature is plotted in Figure ??b, together with its analytical approximation used in the present simulations, which is of the following form:

$$
\operatorname{Pr}(T)=\operatorname{Pr}_{1}\left[1+b_{1}\left(T-T_{1}\right)+b_{2}\left(T-T_{1}\right)^{2}\right]^{-1}
$$

where for water $\operatorname{Pr}_{1}=\operatorname{Pr}\left(T_{1}\right) \simeq 9.47$ and $b_{1} \simeq 3.357 \cdot 10^{-2} \mathrm{~K}^{-1}$ and $b_{2} \simeq 1.884 \cdot 10^{-3} \mathrm{~K}^{-2}$. The resulting flow equations in dimensional form write as follows:

$$
\begin{gathered}
\nabla \cdot \mathbf{v}=0 \\
\frac{\partial \mathbf{v}}{\partial t}+\mathbf{v} \cdot \nabla \mathbf{v}=-\nabla\left(\frac{p}{\rho}\right)+\nabla \cdot\left[\frac{\mu(T)}{\rho}\left(\nabla \mathbf{v}+\nabla^{T} \mathbf{v}\right)\right] \\
\frac{\partial T}{\partial t}+\mathbf{v} \cdot \nabla T=\nabla \cdot\left(\frac{k(T)}{c_{p} \rho} \nabla T\right)+\frac{\Phi}{c_{p} \rho}
\end{gathered}
$$

where $p$ is the pressure and $\Phi=2 \mu(T) \mathbf{S}: \mathbf{S}$ is the dissipation function (we remind that $\nabla \cdot \mathbf{v}=0$ ) and $\mathbf{S}$ the strain rate tensor. Following [?] (see Sec. 7.2 of the referenced book) it is possible to show that, for the problem at issue, we can neglect without any appreciable 
effect on the results the contribution of frictional dissipation in the equations governing the dynamics of temperature, i.e. Equation (??). Indeed, indicating with $U$ and $d$ typical velocity and length scales of the flow and with $\Delta T$ a typical temperature difference, we have:

$$
\frac{\Phi}{c_{p} \rho \frac{D T}{D t}} \sim \frac{\mu}{\rho c_{p}} \frac{U}{\Delta T d}
$$

As an example, for water at $T=20^{\circ} \mathrm{C}$ we have $\mu / \rho c_{p} \simeq 2.4 \cdot 10^{-10} \mathrm{~s} \mathrm{~K}$, and in general for liquids we have $\mu / \rho c_{p} \simeq 10^{-9} \mathrm{~s}$ K. Consequently, the contribution of $\Phi$ to Equation (??) becomes significant only when the term $U /(\Delta T d)$ is very large when considered in compatible dimensional units, which is not the case here. For this reason, from now on we will neglect the dissipation function $\Phi$ in Equation (??).

Equations (??) are made non-dimensional using the hydraulic diameter of the outflow channel, $L_{r}=2 w_{0} h /\left(w_{0}+h\right)$, as the reference length, the bulk velocity $U_{b}$ in the inlet channels as the reference velocity (which is equal to that in the outflow channel for mass conservation) and the temperature $T_{1}$ as the reference temperature. Indicating with the subscript * the non-dimensional quantities, Equations (??) write as:

$$
\begin{gathered}
\nabla_{*} \cdot \mathbf{v}_{*}=0 \\
\frac{\partial \mathbf{v}_{*}}{\partial t_{*}}+\mathbf{v}_{*} \cdot \nabla_{*} \mathbf{v}_{*}=-\nabla_{*} p_{*}+\nabla_{*} \cdot[\underbrace{\frac{f_{R}\left(T_{1}, T_{*}\right)}{\Re_{1}}}_{\alpha\left(T_{*}\right)}\left(\nabla_{*} \mathbf{v}_{*}+\nabla_{*}^{T} \mathbf{v}_{*}\right)] \\
\frac{\partial T_{*}}{\partial t_{*}}+\mathbf{v}_{*} \cdot \nabla_{*} T_{*}=\nabla_{*} \cdot(\underbrace{\frac{f_{R}\left(T_{1}, T_{*}\right) f_{P}\left(T_{1}, T_{*}\right)}{\Re_{1} P r_{1}}}_{\beta\left(T_{*}\right)} \nabla_{*} T_{*})
\end{gathered}
$$

where $\Re_{1}$ and $P r_{1}$ are the Reynolds and Prandtl numbers based on the the flow properties at temperature $T=T_{1}$ and, with reference to Equations (??) and (??):

$$
\begin{aligned}
& f_{R}\left(T_{1}, T_{*}\right)=\left[1+T_{1} a_{1}\left(T_{*}-1\right)+T_{1}^{2} a_{2}\left(T_{*}-1\right)^{2}\right] \\
& f_{P}\left(T_{1}, T_{*}\right)=\left[1+T_{1} b_{1}\left(T_{*}-1\right)+T_{1}^{2} b_{2}\left(T_{*}-1\right)^{2}\right]
\end{aligned}
$$

For the sake of simplicity, in the following we will concisely use the terms $\alpha\left(T_{*}\right)$ and $\beta\left(T_{*}\right)$ so as to replace more complex expressions, as indicated by underbraces in equations (??) 


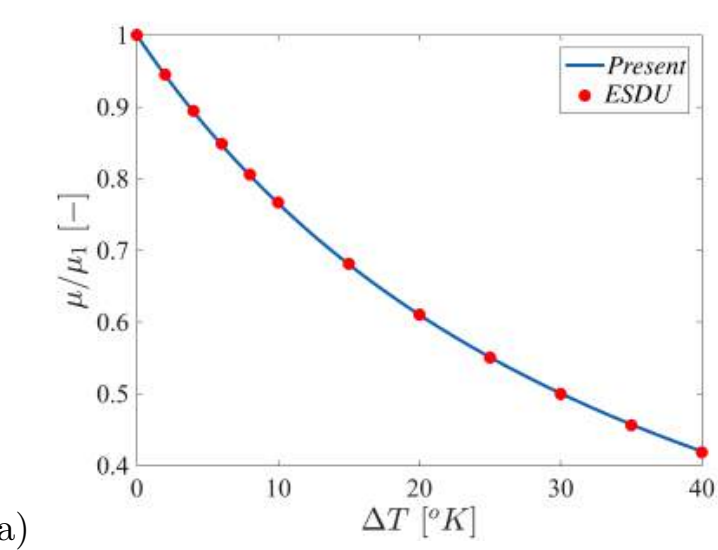

(a)

(b)

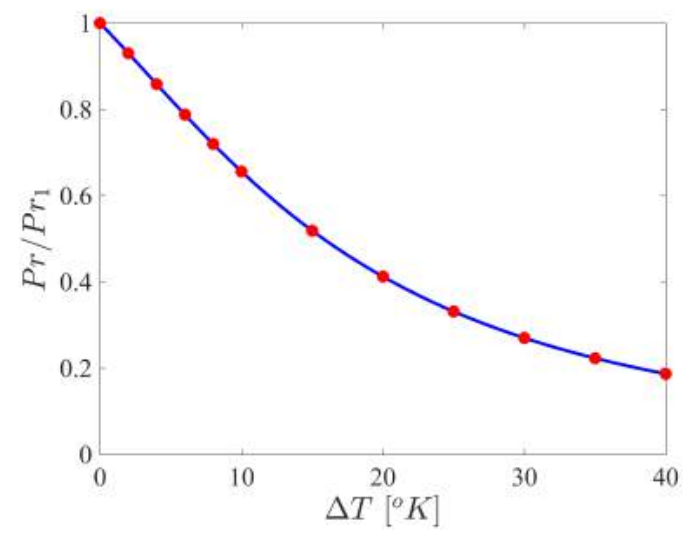

Figure 2. Effect of the variations of temperature on the (a) dynamic viscosity and (b) Prandtl number. Red dots extracted from [? ? ] and corresponding analytical approximation used here

(the dependence of viscosity and diffusivity coefficients on $T_{1}$ is implicitly assumed in the following).

As concerns the boundary conditions for the governing equations (??), at the inflow boundaries both the velocity and the temperature fields are imposed as a Dirichlet boundary condition. The velocity is assumed to have the unidirectional fully developed profile which depends only on the cross section of the inflow channels (see [? ]), while temperature is assumed constant but different on the two inlet channels (equal to $T_{*}=1$ and $T_{*}=T_{2} / T_{1}$, respectively). On the walls homogeneous Dirichlet boundary conditions are imposed for the velocity $\left(\mathbf{v}_{*}=0\right)$ and homogeneous Neumann conditions are imposed on the temperature $\left(\nabla_{*} T_{*} \cdot \mathbf{n}=0\right)$. This last condition, combined with assuming null velocity on the walls, is equivalent to model the walls as adiabatic. Finally, on the outflow boundary we impose stress-free conditions in the momentum equations $\left(\left[p_{*} I-\nabla_{*} \cdot\left(\alpha\left(T_{*}\right)\left(\nabla_{*} \mathbf{V}_{*}+\nabla_{*}^{T} \mathbf{V}_{*}\right)\right)\right] \cdot \mathbf{n}=\right.$ $0)$ and homogeneous Neumann conditions for the temperature field, $\left(\nabla_{*} T_{*} \cdot \mathbf{n}=0\right)$.

\section{STABILITY AND SENSITIVITY ANALYSIS}

Equations (??) are the starting point for the derivation of the stability equations. The stability analysis is applied to a baseflow, $\left(\mathbf{V}_{\mathbf{b} *}, P_{b_{*}}, T_{b_{*}}\right)$ which is a steady solution of equations (??). To this purpose a generic perturbation of the baseflow is searched in modal form 
as follows:

$$
\begin{aligned}
& \mathbf{v}_{*}\left(\mathbf{x}_{*}, t_{*}\right)=\mathbf{V}_{\mathbf{b} *}\left(\mathbf{x}_{*}\right)+\epsilon \hat{\mathbf{v}}_{*}\left(\mathbf{x}_{*}\right) \exp \left(\sigma t_{*}\right) \\
& p_{*}\left(\mathbf{x}_{*}, t_{*}\right)=P_{b *}\left(\mathbf{x}_{*}\right)+\epsilon \hat{p}_{*}\left(\mathbf{x}_{*}\right) \exp \left(\sigma t_{*}\right) \\
& T_{*}\left(\mathbf{x}_{*}, t_{*}\right)=T_{b *}\left(\mathbf{x}_{*}\right)+\epsilon \hat{T}_{*}\left(\mathbf{x}_{*}\right) \exp \left(\sigma t_{*}\right)
\end{aligned}
$$

When the modal form in Equations (??) is substituted in the governing equations (??) and terms are collected depending on the amplitude variable $\epsilon$, at order $\epsilon^{0}$ we find the equations for the baseflow:

$$
\begin{gathered}
\nabla_{*} \cdot \mathbf{V}_{\mathbf{b} *}=0 \\
\mathbf{V}_{\mathbf{b} *} \cdot \nabla_{*} \mathbf{V}_{\mathbf{b} *}=-\nabla_{*} P_{b *}+\nabla_{*} \cdot\left[\alpha\left(T_{b *}\right)\left(\nabla_{*} \mathbf{V}_{\mathbf{b} *}+\nabla_{*}^{T} \mathbf{V}_{\mathbf{b} *}\right)\right] \\
\mathbf{V}_{\mathbf{b} *} \cdot \nabla_{*} T_{b *}=\nabla_{*} \cdot\left(\beta\left(T_{b *}\right) \nabla_{*} T_{b *}\right)
\end{gathered}
$$

At $\operatorname{order} \epsilon$, we find the equations governing the linearised dynamics of the flow perturbations superimposed to the baseflow:

$$
\begin{gathered}
\nabla_{*} \cdot \hat{\mathbf{V}}_{*}=0 \\
\sigma \hat{\mathbf{V}}_{*}+\mathbf{V}_{\mathbf{b} *} \cdot \nabla_{*} \hat{\mathbf{V}}_{*}+\hat{\mathbf{v}}_{*} \cdot \nabla_{*} \mathbf{V}_{\mathbf{b} *}=-\nabla_{*} \hat{p}_{*}+\nabla_{*} \cdot\left[\alpha\left(T_{b *}\right)\left(\nabla_{*} \hat{\mathbf{V}}_{*}+\nabla_{*}^{T} \hat{\mathbf{V}}_{*}\right)\right]+ \\
\nabla_{*} \cdot\left[\left.\frac{\partial \alpha(y)}{\partial y}\right|_{y=T_{b *}}\left(\nabla_{*} \mathbf{V}_{\mathbf{b} *}+\nabla_{*}^{T} \mathbf{V}_{\mathbf{b} *}\right)\right] \hat{T}_{*}
\end{gathered}
$$

$\hat{T}_{*}$

$$
\begin{aligned}
\sigma \hat{T}_{*}+\mathbf{V}_{\mathbf{b} *} \cdot \nabla_{*} \hat{T}_{*}+\hat{\mathbf{v}}_{*} \cdot \nabla_{*} T_{b *}= & \nabla_{*} \cdot\left(\beta\left(T_{b *}\right) \nabla_{*} \hat{T}_{*}\right)+ \\
& \nabla_{*} \cdot\left(\left.\frac{\partial \beta(y)}{\partial y}\right|_{y=T_{b *}} \nabla_{*} T_{b *}\right) \hat{T}_{*}
\end{aligned}
$$

As concerns the boundary conditions for the perturbation, at the inflow boundary both the velocity and the temperature fields are null $\left(\hat{\mathbf{v}}_{*}=0, \hat{T}_{*}=0\right)$. On the walls $\hat{\mathbf{v}}_{*}=0$ and $\nabla_{*} \hat{T}_{*} \cdot \mathbf{n}=0$. On the outflow boundary the following condition applies for the momentum equation:

$$
\left[\hat{p}_{*} I-\nabla_{*} \cdot\left(\alpha\left(T_{b *}\right)\left(\nabla_{*} \hat{\mathbf{V}}_{*}+\nabla_{*}^{T} \hat{\mathbf{V}}_{*}\right)\right)-\nabla_{*} \cdot\left(\left.\frac{\partial \alpha(y)}{\partial y}\right|_{y=T_{b *}}\left(\nabla_{*} \mathbf{V}_{\mathbf{b}_{*}}+\nabla_{*}^{T} \mathbf{V}_{\mathbf{b} *}\right)\right) \hat{T}_{*}\right] \cdot \mathbf{n}=0
$$

and, for the temperature equation, we have $\nabla_{*} \hat{T}_{*} \cdot \mathbf{n}=0$. 
Equations (??), together with the boundary conditions discussed above, form an eigenfunction problem with generally complex eigenvalues $\sigma=\lambda+\mathrm{i} \omega$. The baseflow $\left(\mathbf{V}_{\mathbf{b} *}, P_{b *}, T_{b *}\right)$ is stable if all eigenvalues $\sigma$ have negative real part. Conversely, an unstable mode is identified if the associated eigenvalue has a postive real part $(\lambda>0)$.

Let us suppose that one particular unstable eigenmode is identified, $\left(\hat{\mathbf{v}}_{*}, \hat{p}_{*}, \hat{T}_{*}\right)$, associated to the eigenvalue $\sigma$. Generalizing the work in [?], we want to investigate the sensitivity of the eigenvalue $\sigma$ to a generic structural perturbation of the linearised equations (??). In particular, we consider two localised types of perturbations, acting on the momentum and energy equations, respectively. As concerns the momentum equation, we assume a localised velocity-force feedback of the following form:

$$
\delta \mathbf{f}\left(\mathbf{x}_{*}\right)=\left[\mathbf{M}_{\mathbf{0}} \cdot \hat{\mathbf{v}}_{*}\left(\mathbf{x}_{*}\right)\right] \delta\left(\mathbf{x}_{*}-\mathbf{x}_{* 0}\right)
$$

where $\mathbf{M}_{\mathbf{0}}$ is a constant generic feedback matrix, $\delta(\cdot)$ is the Dirac delta function and $\mathbf{x}_{* 0}$ is the generic point of application of the local forcing. Analogously, we investigate a structural perturbation of the temperature equation of the following form:

$$
\delta g\left(\mathbf{x}_{*}\right)=\left[K_{0} \hat{T}_{*}\left(\mathbf{x}_{*}\right)\right] \delta\left(\mathbf{x}_{*}-\mathbf{x}_{* 0}\right)
$$

where $K_{0}$ is a generic feedback constant. A conceptually similar structural perturbation has been considered in [? ] for quantifying the effect of a localised heat source in a compressible flow. In order to investigate the effect of the two structural perturbations introduced above on the eigenvalue $\sigma$, so as to derive the resulting eigenvalue perturbation $\delta \sigma$, it is convenient to derive the adjoint equations of the system (??). The derivation is a classical application of calculus of variations (see Appendix ?? for details), and the resulting system of equations in terms of the adjoint variables $\left(\tilde{\mathbf{v}}_{*}\left(\mathbf{x}_{*}\right), \tilde{p}_{*}\left(\mathbf{x}_{*}\right), \tilde{T}_{*}\left(\mathbf{x}_{*}\right)\right)$ writes as follows:

$$
\begin{gathered}
\nabla_{*} \cdot \tilde{\mathbf{v}}_{*}=0 \\
\sigma^{*} \tilde{\mathbf{V}}_{*}-\mathbf{V}_{\mathbf{b} *} \cdot \nabla_{*} \tilde{\mathbf{V}}_{*}+\tilde{\mathbf{v}}_{*} \cdot \nabla_{*} \mathbf{V}_{\mathbf{b} *}=-\nabla_{*} \tilde{p}_{*}+\nabla_{*} \cdot\left[\alpha\left(T_{b *}\right)\left(\nabla_{*} \tilde{\mathbf{V}}_{*}+\nabla_{*}^{T} \tilde{\mathbf{V}}_{*}\right)\right]- \\
\left(\nabla_{*} T_{b *}\right) \tilde{T}_{*} \\
\sigma^{*} \tilde{T}_{*}-\mathbf{V}_{\mathbf{b} *} \cdot \nabla_{*} \tilde{T}_{*}+\frac{1}{2}\left(\nabla_{*} \tilde{\mathbf{V}}_{*}+\nabla_{*}^{T} \tilde{\mathbf{V}}_{*}\right):\left.\left(\nabla_{*} \mathbf{V}_{\mathbf{b} *}+\nabla_{*}^{T} \mathbf{V}_{\mathbf{b} *}\right) \frac{\partial \alpha(y)}{\partial y}\right|_{y=T_{b *}}= \\
=-\left.\frac{\partial \beta(y)}{\partial y}\right|_{y=T_{b *}} \nabla_{*} \tilde{T}_{*} \cdot \nabla_{*} T_{b *}+\nabla_{*} \cdot\left(\beta\left(T_{b *}\right) \tilde{T}_{*}\right)
\end{gathered}
$$


As for the boundary conditions, at the inflow we have $\tilde{\mathbf{v}}_{*}=\mathbf{0}$ and $\tilde{T}_{*}=0$, at the walls $\tilde{\mathbf{v}}_{*}=\mathbf{0}$ and $\nabla_{*} \tilde{T}_{*} \cdot \mathbf{n}=0$. Finally, on the outflow boundary for the momentum equations we have:

$$
-\tilde{p}_{*} \mathbf{n}+\left(\mathbf{V}_{\mathbf{b} *} \cdot \mathbf{n}\right) \tilde{\mathbf{V}}_{*}+\alpha\left(T_{b *}\right)\left(\nabla_{*} \tilde{\mathbf{V}}_{*}+\nabla_{*}^{T} \tilde{\mathbf{V}}_{*}\right) \cdot \mathbf{n}=\mathbf{0}
$$

and for the energy equation we have:

$$
\left(\mathbf{V}_{\mathbf{b} *} \cdot \mathbf{n}\right) \tilde{T}_{*}+\beta\left(T_{b *}\right) \nabla_{*} \tilde{T}_{*} \cdot \mathbf{n}=0
$$

The direct and adjoint modes obtained from equations (??) and equations (??) are successively normalised as follows:

$$
\begin{aligned}
& \left\langle\hat{\mathbf{v}}_{*}, \tilde{\mathbf{v}}_{*}\right\rangle=\int_{\Omega}\left(\hat{\mathbf{v}}_{*}^{*} \cdot \tilde{\mathbf{v}}_{*}\right) \mathrm{d} \Omega=1 \\
& \left\langle\hat{T}_{*}, \tilde{T}_{*}\right\rangle=\int_{\Omega}\left(\hat{T}_{*}^{*} \tilde{T}_{*}\right) \mathrm{d} \Omega=1,
\end{aligned}
$$

denoting with the symbol ${ }^{*}$ the conjugate of a complex quantity. Using the same variational approach leading to the adjoint equations, it is possible to show that the variation $\delta \sigma$ of the eigenvalue $\sigma$ caused by the structural perturbations $\delta \mathbf{f}$ and $\delta g$ (see equation (??) and equation (??), respectively) is given by the following equation

$$
\delta \sigma=\left\langle\tilde{\mathbf{v}}_{*}, \delta \mathbf{f}\left(\mathbf{x}_{*}\right) \hat{\mathbf{v}}_{*}\right\rangle+\left\langle\tilde{T}_{*}, \delta g\left(\mathbf{x}_{*}\right) \hat{T}_{*}\right\rangle
$$

Using the properties of the Dirac function and the triangular inequality, the variation $\delta \sigma$ can be bounded as follows:

$$
|\delta \sigma| \leq\left\|\mathbf{M}_{\mathbf{0}}\right\| \underbrace{\left\|\tilde{\mathbf{v}}_{*}\left(\mathbf{x}_{*}\right)\right\|\left\|\hat{\mathbf{v}}_{*}\left(\mathbf{x}_{*}\right)\right\|}_{S_{M}\left(\mathbf{x}_{*}\right)}+\left|K_{0}\right| \underbrace{\left|\hat{T}_{*}\left(\mathbf{x}_{*}\right)\right|\left|\tilde{T}_{*}\left(\mathbf{x}_{*}\right)\right|}_{S_{T}\left(\mathbf{x}_{*}\right)}
$$

where $\|\cdot\|$ is a generic vector norm and the corresponding induced matrix norm and $|\cdot|$ is the absolute value. Looking at the inequality (??) it is possible to deduce that the scalar maps $S_{M}\left(\mathbf{x}_{*}\right)$ and $S_{T}\left(\mathbf{x}_{*}\right)$ are positive-definite sensitivity maps for the structural perturbation of the momentum and temperature equations, respectively. The maps, depending on space $\mathbf{x}_{*}$, are proportional to the effect on $\delta \sigma$ consequent to introducing locally at position $\mathbf{x}_{*}$ the considered structural perturbations $\delta \mathbf{f}$ and $\delta g$. In particular, following [? ], regions where the sensitivity map is not negligibly small indicate the zone where the considered structural perturbation has a significant influence on the considered eigenvalue. When this 
region is bounded and localised in space, it can be identified as the core of the instability (see [? ] and [? ] for more details). Here, at difference with the classical case of the Navier Stokes equations for an incompressible flow with constant fluid properties, we have two kind of sensitivities associated to the structural perturbation of the momentum and temperature equations, respectively. Their shape in space for the engulfment instability and the discussion of the related properties is postponed in section ??

\section{NUMERICAL METHODS AND CONVERGENCE TESTS}

\section{A. Direct numerical simulations}

The DNSs are carried out by discretizing Eqs. (??) using the open-source code Nek5000 (https://nek5000.mcs.anl.gov). It is a massively parallel code based on a spectralelement method (SEM), where the spatial discretisation of the domain is carried out using hexahedral elements. The unknown variables are spanned by Nth-order Lagrange polynomial interpolants, based on tensor-product arrays of Gauss-Lobatto-Legendre (GLL) quadrature points in each spectral element, where typically $N \geq 6$. The time discretisation of the system (??) is obtained employing a third-order backward differentiation formula (BDF3). As concerns the spatial discretisation, the diffusive terms are treated implicitly, while a third order explicit extrapolation formula (EXT3) is considered for the convective terms. The explicit extrapolations for the convective terms in the BDF3-EXT3 scheme impose a restriction of the time-step, $\Delta \mathrm{t}$, (see for instance [? ]) for iterative stability. For this reason $\Delta \mathrm{t}$ is chosen here so as to have a Courant number equal to $\mathrm{CFL} \approx 0.5$.

The computational domain adopted for the DNSs has the same characteristics as the one validated and used in [? ]. With reference to figure ??, $L_{i}=6.25 L_{r}$ and $L_{o}=25 L_{r}$ are chosen as length of the inlet and outlet channels, respectively. This domain is discretised through a structured multi-block grid having 15680 spectral elements; the grid used herein is the same as the one in [? ], to which we refer for more details. After a number of prefinement tests (not shown here for sake of brevity), the polynomial order for the velocity components and for the temperature is fixed at $N=7$, while order 5 is selected for the pressure, using the $P_{N}-P_{N-2}$ approach for the elimination of spurious pressure modes. 


\section{B. Global stability analysis}

The three-dimensional global stability analysis is performed using the finite element code FreeFem $++($ http: //www.freefem.org). First, the baseflow is interpolated on a structured grid suitable for the finite element solver (see ??b) and, successively, the global eigenvalue problem (??) is solved using the parallel iterative solver HIPS ( Hierarchical Iterative Parallel Solver, http://hips.gforge.inria.fr). This scientific library is suitable for large sparse problems and implements a type of multilevel ILU as preconditioner (see [? ]). In order to study how the dimensions of the computational domain and the mesh refinement affect the results of the global stability analysis, different grids have been tested. We remind that these grids are used only for the stability analysis, while flow simulation providing the baseflow is carried out on a independent grid using Nek5000. The results of this convergence test are reported for the case at $\Delta T=40^{\circ} \mathrm{C}$ and $R e=105$ in table ??. In particular, with reference to figure ??b, the table details the characteristics of the considered grids and computational domains together with the corresponding leading eigenvalues obtained by stability analysis. The stability analysis performed on the meshes $\mathrm{C} 1$ and $\mathrm{C} 2$ shows that the position of the outlet boundary (defined by the $y$-coordinate $y_{1}$ ) has a negligible impact on the evaluated leading eigenvalue, at least for the two lengths of the outlet channel considered. Conversely, the position of the inlet boundaries (defined by the coordinate $x_{2}$ ) has a strong influence on the evaluation of the global eigenfrequency, as it is clear comparing the results on the grid $\mathrm{C} 1$ and C3. Finally, starting from C2, progressively refined grids have been tested for the same computational domain (from $\mathrm{C} 4$ to $\mathrm{C} 7$ ). Comparing the results from $\mathrm{C} 6$ and $\mathrm{C} 7$, a small impact on the resulting real eigenvalue is found showing grid convergence. Thus, in the following, all the presented global stability results are obtained using the mesh C6.

\section{RESULTS}

\section{A. Direct numerical simulations}

A first characterisation of the vortex and engulfment regimes has been carried out by performing several DNS simulations. For different values of the temperature difference of the two streams entering the mixer, i.e. $\Delta T=T_{2}-T_{1}$, different values of the flow Reynolds

numbers $\Re_{1}$ have been considered so as to detect the critical value $R e_{c r}$ for the occurrence 


\begin{tabular}{ccccccccc} 
Mesh & $x_{2}$ & $y_{2}$ & $n_{e}$ & $n_{v}$ & $n_{\text {dof }}$ & $h_{\max }$ & $h_{\min }$ & $10^{3} \lambda_{0}$ \\
\hline C1 & 3.0 & -10.0 & 176832 & 33891 & 1046391 & 0.438 & 0.039 & -12.388 \\
C2 & 3.0 & -3.0 & 82560 & 16467 & 499047 & 0.441 & 0.039 & -14.711 \\
C3 & 1.5 & -3.0 & 97920 & 19019 & 583519 & 0.197 & 0.039 & +2.868 \\
C4 & 3.0 & -3.0 & 135360 & 26169 & 804669 & 0.438 & 0.036 & -13.613 \\
C5 & 3.0 & -3.0 & 180768 & 34245 & 1063049 & 0.451 & 0.035 & -9.194 \\
C6 & 3.0 & -3.0 & 201600 & 38311 & 1187611 & 0.435 & 0.031 & -6.561 \\
C7 & 3.0 & -3.0 & 228096 & 42670 & 1332442 & 0.459 & 0.034 & -6.728
\end{tabular}

Table I. Results on convergence tests of the global stability analysis at $R e=105$ and $\Delta T=40^{\circ} \mathrm{C}$; $x_{2}$ and $y_{2}$ are defined in figure $? ? \mathrm{~b} ; n_{e}$ and $n_{v}$ are the numbers of elements and vertices of the mesh; $n_{d o f}$ is the resulting number of degrees of freedom for the unknown variables $[u, v, w, \theta, p] ; h_{\max }$ and $h_{\min }$ are the resulting maximum and minimum grid spacing, respectively; $\lambda_{0}$ is the leading global eigenvalue obtained by stability analysis.

of the engulfment bifurcation. The different flow regimes (vortex or engulfment regime) identified at different values of $\Re_{1}$ are summarised in Figure ?? for each $\Delta T$ here considered. These results show that $R e_{c r}$ decreases as $\Delta T$ is increased, reaching an almost constant value with $\Delta T$ for $\Delta T \geq 30^{\circ} \mathrm{C}$. Quantitatively, while $135 \leq R e_{c r} \leq 145$ when $\Delta T=0^{\circ} \mathrm{C}$, the $R e_{c r}$ reaches the value of 115 for $\Delta T=30^{\circ} \mathrm{C}$. Lastly, when $\Delta T$ is greater than $30^{\circ} \mathrm{C}$, the effect of the temperature variation on the $R e_{c r}$ can be considered mild, as it is clearly shown in figure ??.

The engulfment or vortex regimes have been identified in figure ?? by performing a simulation for each reported point and by checking the symmetries of the resulting steady solution. We remind that both the vortex and the engulfment regimes are steady and, depending on the saturation velocity of the instability, engulfment regime can take long time to settle especially for Reynolds numbers close to the critical one.

Besides visual inspection of the flow symmetries, a complementary way to highlight the development of the engulfment regime is by inspecting the mixing degree between the two flows entering the two inlet channels, since it is known that engulfment is associated with a rapid increase of mixing (see for instance [?]). In order to quantify mixing, a non- 


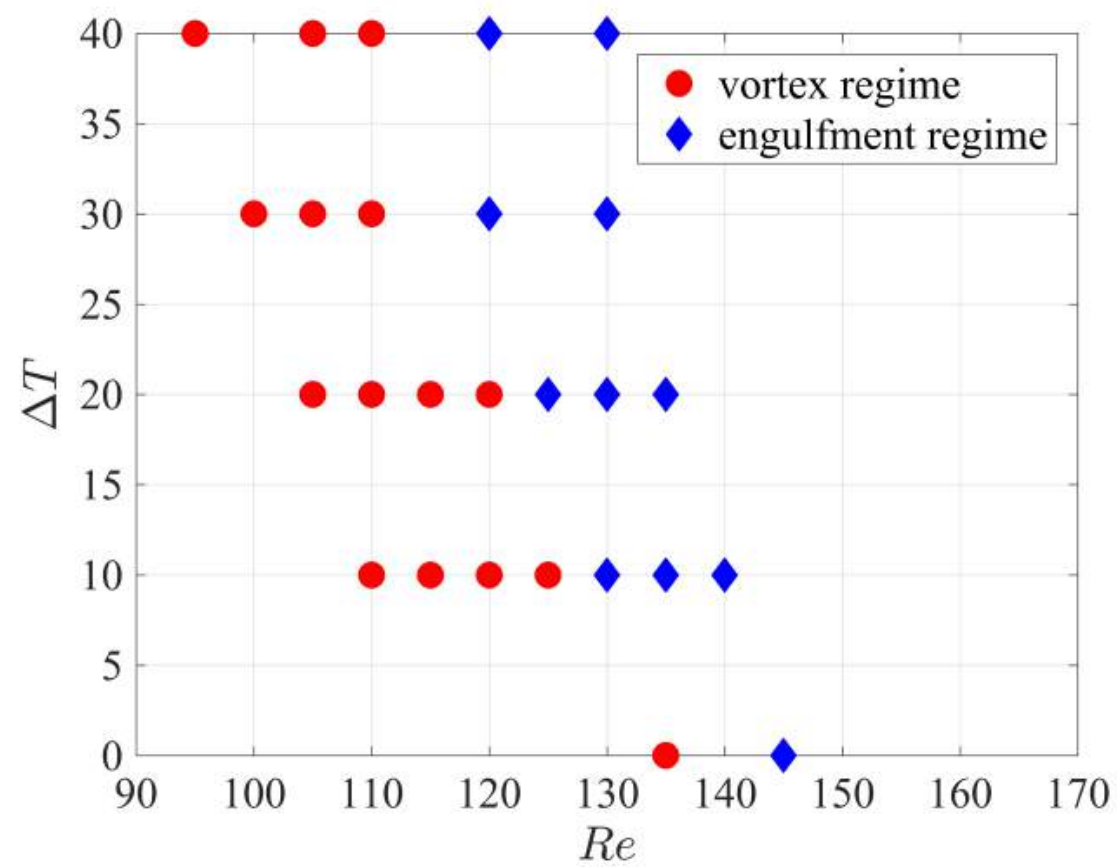

Figure 3. Flow regimes (vortex or engulfment regime) identified using DNSs for different values of the Reynolds number $\Re_{1}$ at different values of the temperature jump between the two streams entering the T-mixer $\Delta T$.

dimensional quantity $\phi$ is defined:

$$
\phi(x, y, z)=\frac{T(x, y, z)-T_{1}}{\Delta T}
$$

Following [? ] and using the definition in Eq. (??), the mixing degree $\delta_{m}$ in the outlet channel has been defined as follows:

$$
\delta_{m}(y)=1-\frac{\sigma_{b}(y)}{\sigma_{\max }}
$$

where the bulk value of the quantity $\bar{\phi}_{b}$ and the standard deviation of the volumetric flow $\sigma_{b}$ are defined as follows:

$$
\begin{gathered}
\bar{\phi}_{b}=\frac{\int \phi(x, y, z) V(x, y, z) d x d z}{\int V(x, y, z) d x d z} \\
\sigma_{b}^{2}(y)=\frac{\int\left(\phi(x, y, z)-\bar{\phi}_{b}\right)^{2} V(x, y, z) d x d z}{\int V(x, y, z) d x d z}
\end{gathered}
$$

where $V(x, y, z)$ is the $y$-velocity component in the $y$ cross section within the outlet channel. $\sigma_{\max }$ is the maximum value of $\sigma_{b}$, which is achieved when the two streams remain completely 


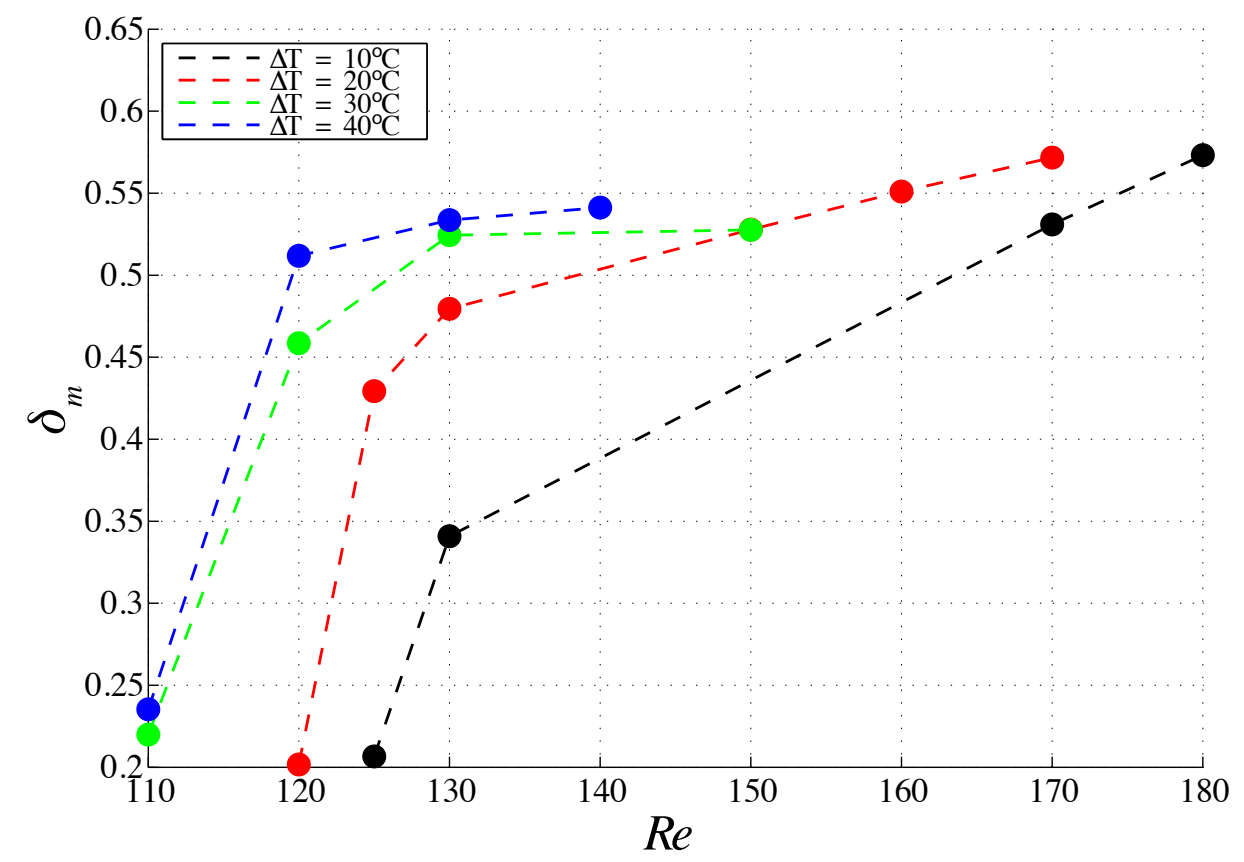

Figure 4. Degree of mixing $\delta_{m}$ evaluated in the outlet channel at $\mathrm{y}=-5$ for different temperature jumps.

segregated, and it is defined as:

$$
\sigma_{\max }=\sqrt{\bar{\phi}_{b}\left(1-\bar{\phi}_{b}\right)}
$$

As a result, $\delta_{m}$ is bounded in the range $0 \leq \delta_{m} \leq 1$, and $\delta_{m}=0$ and $\delta_{m}=1$ indicate a completely segregated and a fully mixed flow, respectively. The mixing parameter $\delta_{m}$ has been computed using the available simulations and the results are reported in figure ??. Inspecting figure ?? it is possible to notice that, for each temperature jump $\Delta T$, there is a sudden increase of mixing as the engulfment regime develops, i.e. as Re becomes larger than $R e_{c r}$. For this reason it is possible to deduce an approximate value of $R e_{c r}$ looking at the behaviour of mixing in the referenced figure. In particular, figure ?? confirms that $R e_{c r}$ is almost constant for $\Delta T \geq 30^{\circ} \mathrm{C}$, as also deduced from figure ??. Moreover, it can be evinced that the increase of mixing is progressively sharper with the flow Reynolds number when $\Delta T$ is increased. For instance, at $\Delta T=10^{\circ} \mathrm{C}$, the value of $\delta_{m}$ starts to increase starting from $R e \simeq 130$, but it does not reach an asymptotic value in the range of considered Reynolds numbers $(R e \leq 180)$. Conversely, $\delta_{m}$ starts to increase at $R e=110$ for $\Delta T=40^{\circ} \mathrm{C}$ and it reaches an asymptotic value of $\delta_{m} \simeq 0.55$ for $R e \simeq 130$. This behaviour indicates that, as $\Delta T$ is increased, the engulfment instability increases more sharply with $R e$. 
As concerns the characteristics of the flow fields in the vortex and the engulfment regimes, we now discuss the main differences that can be observed by comparison between the case with the highest temperature difference, $\Delta T=40^{\circ} \mathrm{C}$ (hereafter denoted DT40) and the case without temperature difference (denoted DT0), which has been widely investigated in the literature. In particular, in [? ? ] the same T-mixer configuration was analysed for $\Delta T=0$ both through DNS and stability analysis.

In figure ?? representative isosurfaces of the $\lambda_{2}$ field are reported, $\lambda_{2}$ being the scalar parameter in the vortex identification criterion described in [? ], so as to identify the vortical structures of the flow in the vortex regime. The two flow fields in figure ?? have been taken at a Reynolds number which is slightly subcritical for the engulfment instability, which is equal to $R e=135$ and $R e=110$ for the cases DT0 and DT40, respectively. As for the DT0 case, depicted in figure ??(a), also in the case DT40 (see figure ??(b)) two vortices originate at the confluence region between the two incoming streams. The vortices have their axis parallel to the $z$ direction and they extend towards the outflow channel with two "legs" which remain almost parallel to the $y$ direction and in specular position with respect to plane $z=h / 2$. As a result, at each $x z$ section in the outflow channel we can identify 4 vortices whose axis is almost parallel to the $y$ direction, two rotating in clockwise and two in counter-clockwise direction. In the DT0 case the flow field has two reflectional symmetries, with respect to the $z=h / 2$ and to the $x=0$ planes. Conversely, for the DT40 case, and in general when $\Delta T \neq 0^{\circ} \mathrm{C}$, the flow is no more symmetric with respect to the plane $x=0$. This is already evident by the asymmetry in the shape of the two couples of vortices located in the outlet channel which can be observed in figure ??(b).

For a more detailed comparison, the distribution of temperature is shown in figure ?? at selected $x z$ sections for five different values of $\Delta T$, from $\Delta T=0^{\circ} \mathrm{C}$ to $\Delta T=40^{\circ} \mathrm{C}$. In the case of $\Delta T=0^{\circ} \mathrm{C}$, the distribution of the concentration of a passive scalar is shown for comparison with temperature. In figure 6 and in the following, the range of variation of the passive tracer is arbitrarily fixed between 10 and 11 to make the comparison with the distribution of $\mathrm{T}$ easier.Figure ?? comprises 5 different values of $\Delta T$, but for the moment we focus only on DT0 and DT40 only. For each value of $\Delta T$, the flow Reynolds number considered is just subcritical for the engulfment instability so that all cases have approximately the same "distance" in terms of Re from the instability. Let us focus for the moment only on the DT0 and DT40 cases only. By comparison between the passive tracer in DT0 and temperature 
field for DT40 the loss of one reflectional symmetry is evident. As a consequence, since the viscosity depends on temperature and thus couples the momentum and the temperature equations, also the velocity field looses the reflectional symmetry with respect to the plane $x=0$, as highlighted in Figure ??. Figure ?? also shows another interesting behaviour which is caused by the variation of viscosity with temperature. Colder fluid, which implies higher viscosity for water, passes to the hotter half of the mixer $(x \geq 0)$ flowing in a complex way in the confluence region between the two incoming streams. A more precise idea of the temperature distribution in this region can be gained integrating the views in Figure ?? with those in Figure ??, where the temperature distribution at selected $x y$ sections is reported, with focus on the confluence region. The complex asymmetrical behaviour described above can be guessed also from Figure ??, where it can be noticed that the vortex forming in the cold region (blue vortex) is slightly higher in the $y$ direction than the hot one. The cold fluid passing to the other half of the mixer in the confluence region descend the outlet channel staying adjacent to the wall $x=w_{0} / 2$, thus creating a colder layer which can be clearly seen in Figure ?? for the section $y=-1$.

Due to the coupling between temperature and velocity through viscosity, the temperature difference $\Delta T$ has also a significant impact on the velocity and vorticity distribution. In particular, looking again at Figure ?? it can be noticed that the two vortices in the colder region dissipates more quickly than the two equivalent ones in the hotter region, viscosity in the colder fluid being larger by a factor approximately equal to 5 for DT40. This fact further increases the flow asymmetry and explains the particular temperature distribution at $y=-3$ that can be observed in Figure ??, where hotter water penetrates in the cold half of the mixer $(x \leq 0)$ especially in proximity of the lateral walls $(x= \pm h / 2)$. This loss of symmetry leads, for the largest values of $\Delta T$, to a small amount of mixing also in the vortical regime (see figure 4). Since, as it will be discussed in the following, the engulfment regime takes place when the flow symmetries are broken, the previous observations are also consistent with the fact that increasing $\Delta T$ the value of the critical Reynolds number decreases.

In the cases with a value of $\Delta T$ which is intermediate between DT0 and DT40, also reported in Figures ?? and ??, the flow behaviour is regularly changing with $\Delta T$ and is thus intermediate between the DT0 and DT40 cases. For this reason we will not comment them further.

With reference to Figure ??, the onset of the engulfment regime leads to a modification 

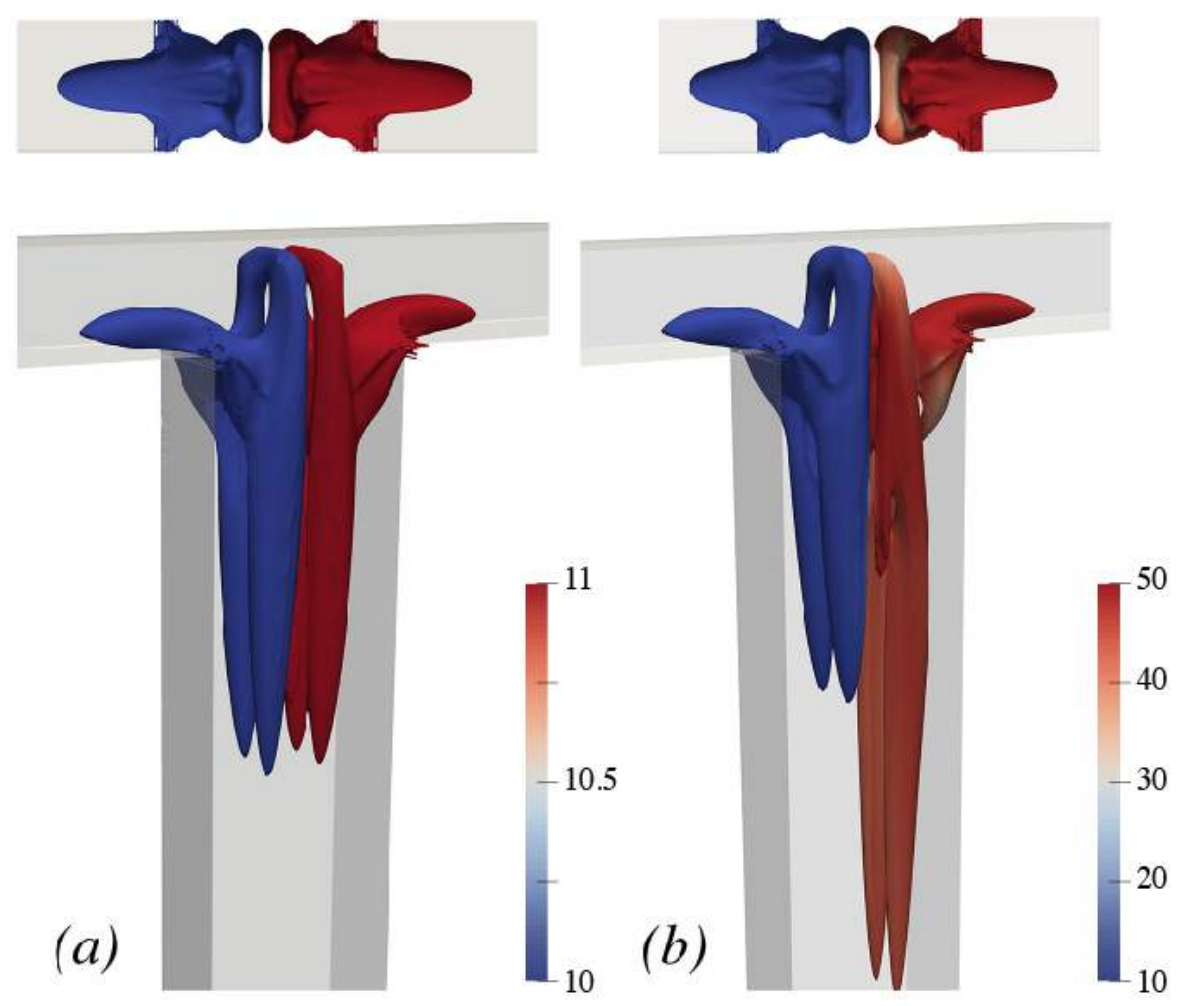

Figure 5. Top and lateral views of the $3 \mathrm{D}$ vortical structures identified by the $\lambda 2$ criterion ([? ]) for the vortex regime at (a) $\Delta T=0^{\circ} \mathrm{C}$ and $R e=135$ and (b) $\Delta T=40^{\circ} \mathrm{C}$ and $R e=110$.

of the flow which is similar to what observed in the literature in the DT0 case. In particular, the two vortices forming near the top wall of the mixer $(y=w)$ tilt their axis in the $y z$ plane, thus breaking the reflectional symmetry with respect to the plane $z=0$, which is the only reflectional symmetry that can be found in the vortex regime when $\Delta T \neq 0$. As a consequence, the two legs of each vortex, which enters the outflow channel due to convection, are asymmetric and their intensity is different. While in the case DT0 the flow in the engulfment regime preserves a single reflectional symmetry, all symmetries are lost when $\Delta T \neq 0^{\circ} \mathrm{C}$. In all cases, however, only two co-rotating vortices remain in the outlet conduit at a sufficient distance from the confluence region of the three channels, and their induced velocity field is the main characteristic causing, by convection, the increase of mixing observed in this flow regime. The fact that the intensity of these two surviving vortices is slightly different when $\Delta T \neq 0^{\circ} \mathrm{C}$ does not alter significantly the scenario and the characteristics of the resulting flow, if compared to the DT0 case, as shown in figure ?? and figure ?? where the temperature and the velocity fields for the engulfment regime at 
$\Delta T=0^{\circ} C, R e=135$
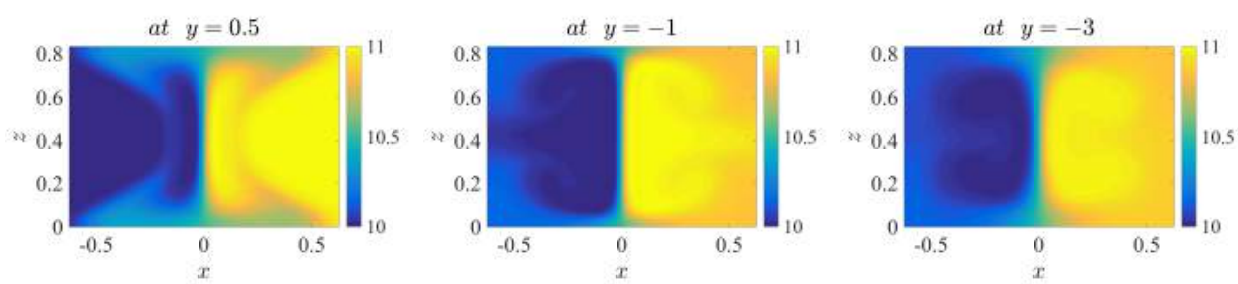

$$
\Delta T=10^{\circ} C, R e=120
$$
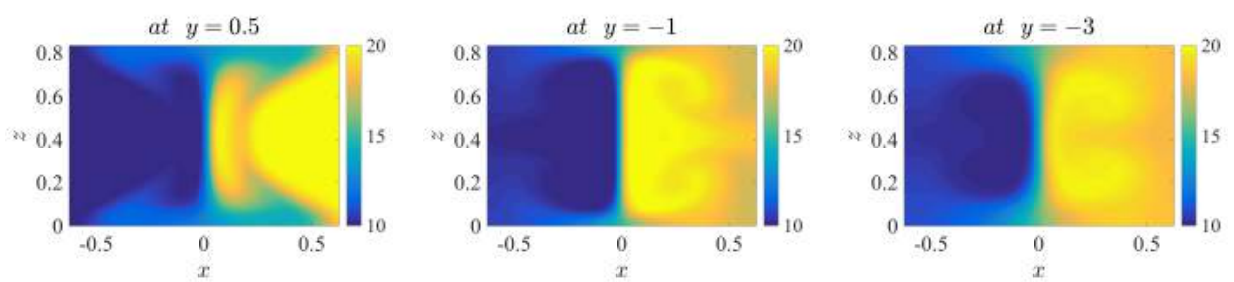

$\Delta T=20^{\circ} C, R e=115$
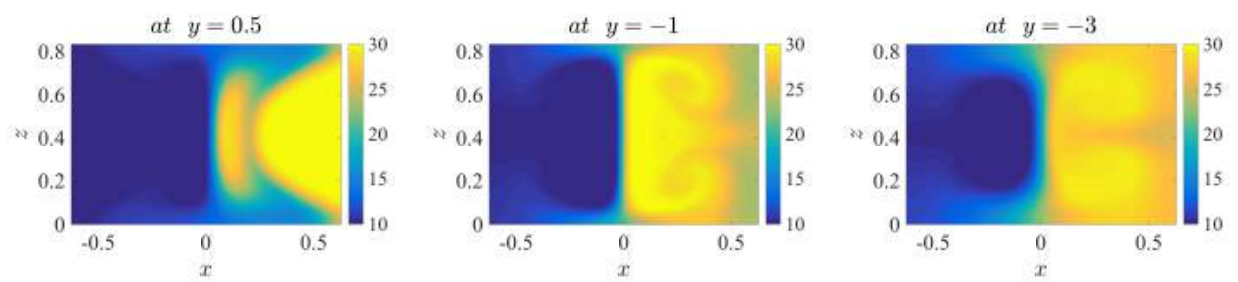

$\Delta T=30^{\circ} C, R e=110$
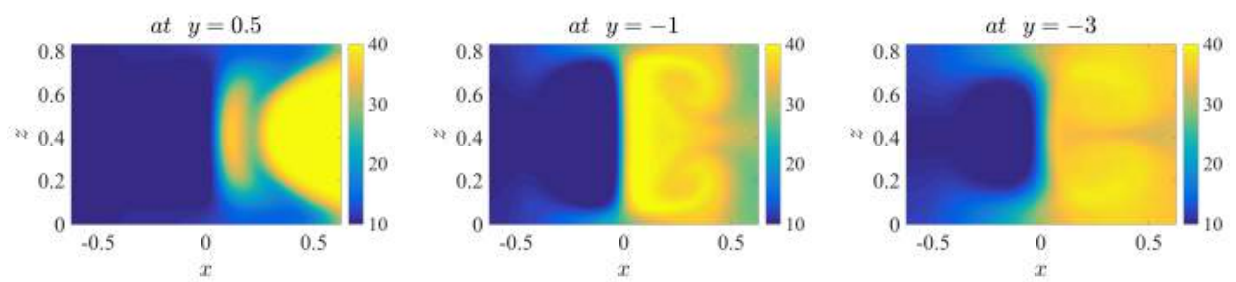

$$
\Delta T=40^{\circ} C, R e=110
$$
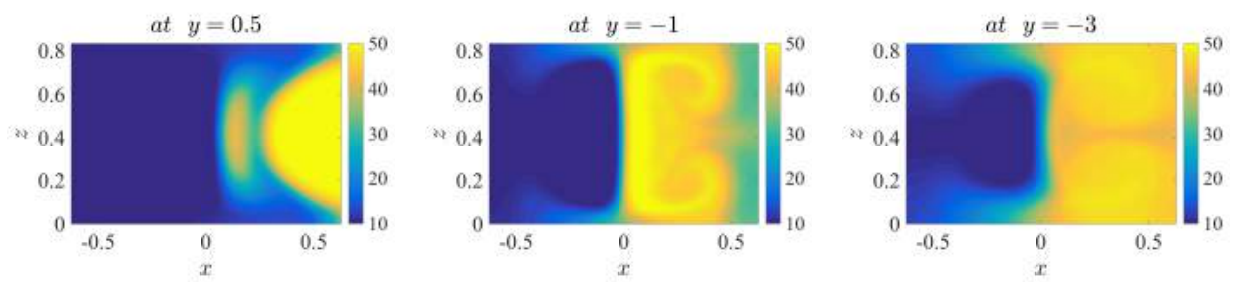

Figure 6. Distribution of temperature in the vortex regime for different values of the temperature jump $\Delta T=T_{2}-T_{1}$ at three selected sections in $x z$-plane at $y=0.5,-1,-3$ 
$\Delta T=0^{\circ} C, R e=135$
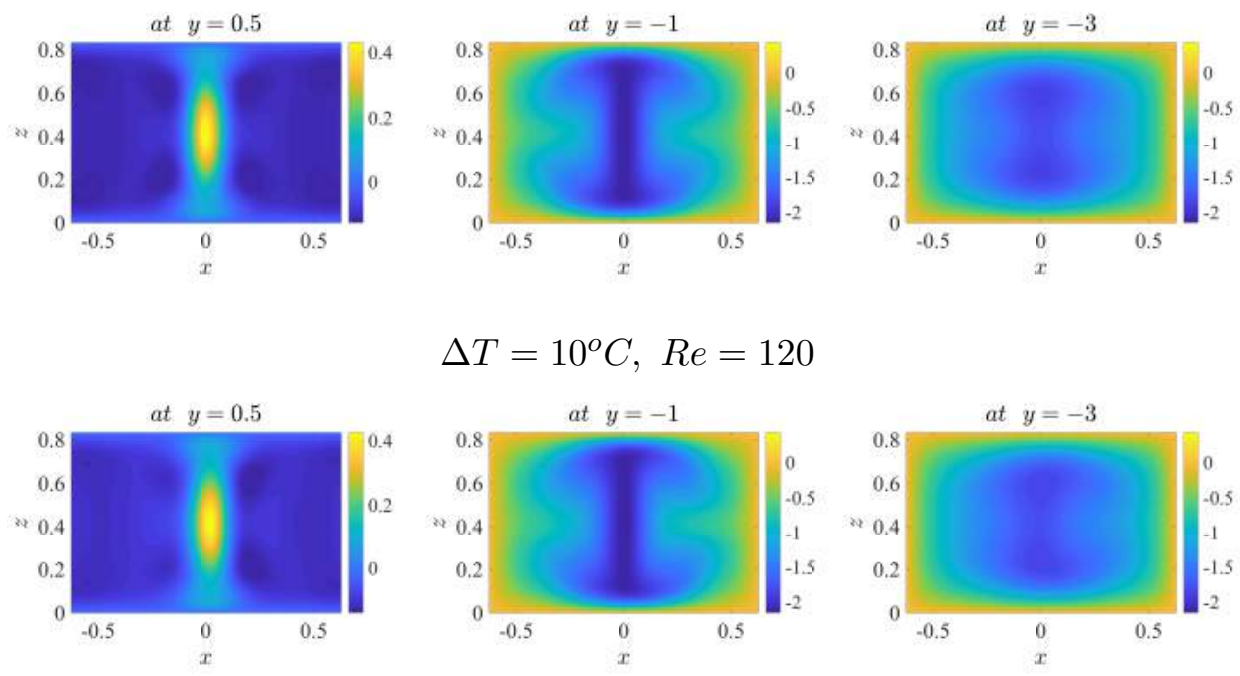

$\Delta T=10^{\circ} C, R e=120$
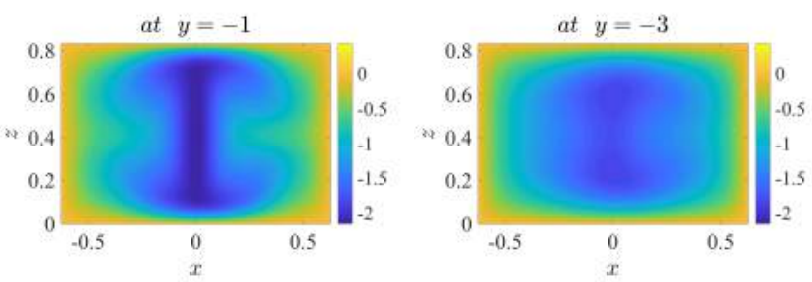

$$
\Delta T=20^{\circ} C, R e=115
$$
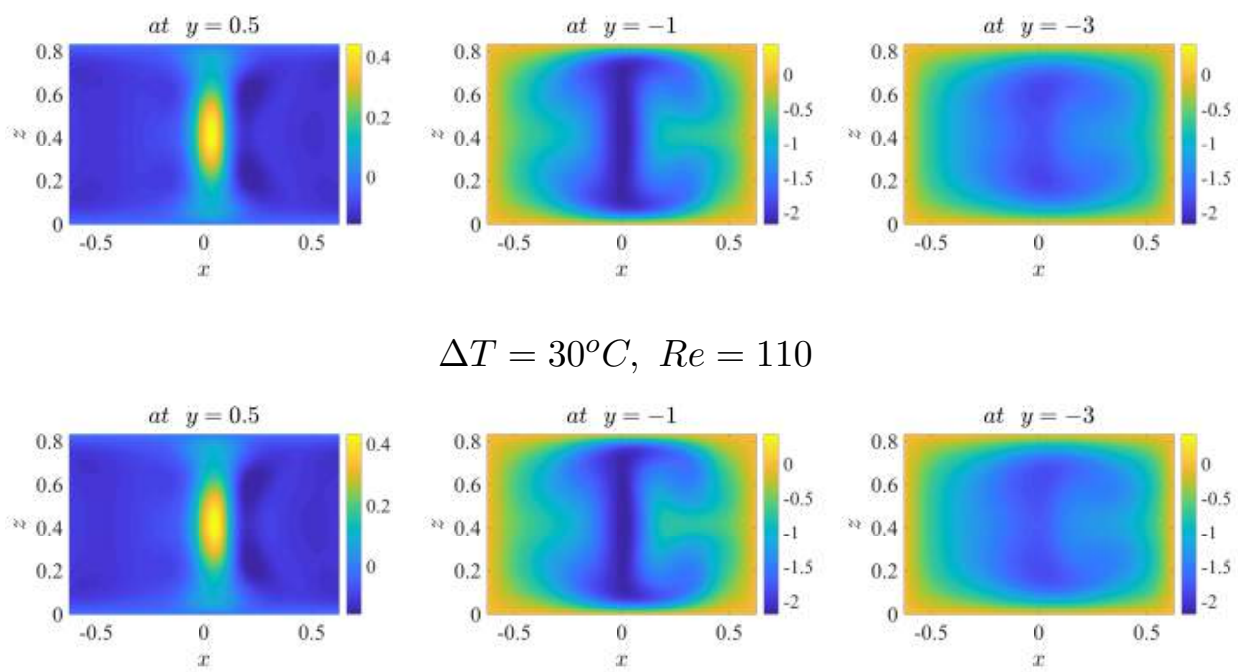

$$
\Delta T=30^{\circ} C, R e=110
$$
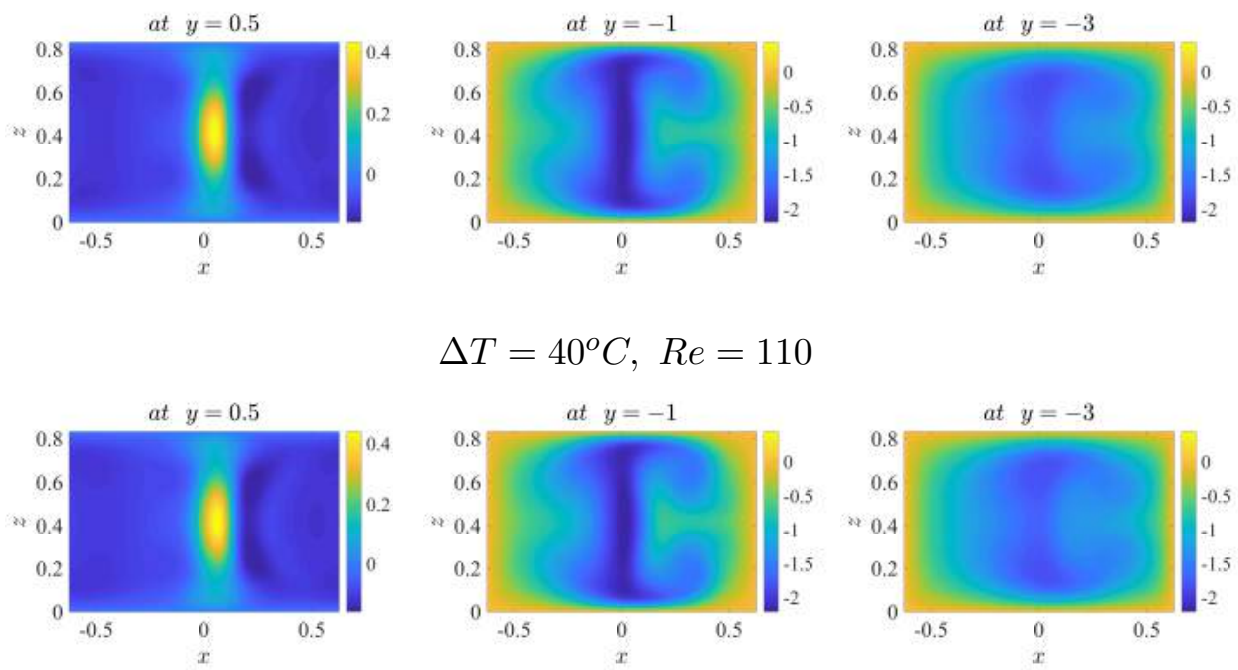

$$
\Delta T=40^{\circ} C, R e=110
$$
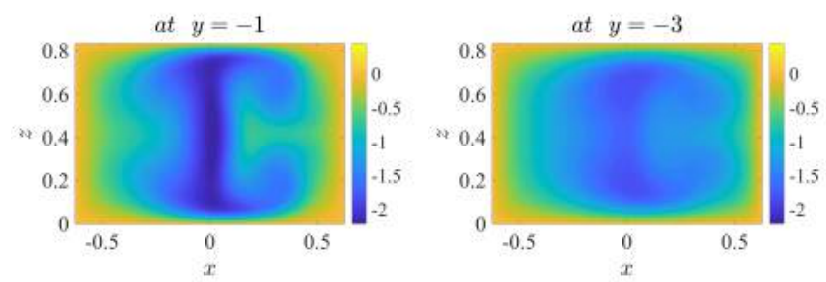

Figure 7. Distribution of the velocity in $y$ direction in the vortex regime for different values of the temperature jump $\Delta T=T_{2}-T_{1}$ at three selected sections in $x z$-plane at $y=0.5,-1,-3$ 

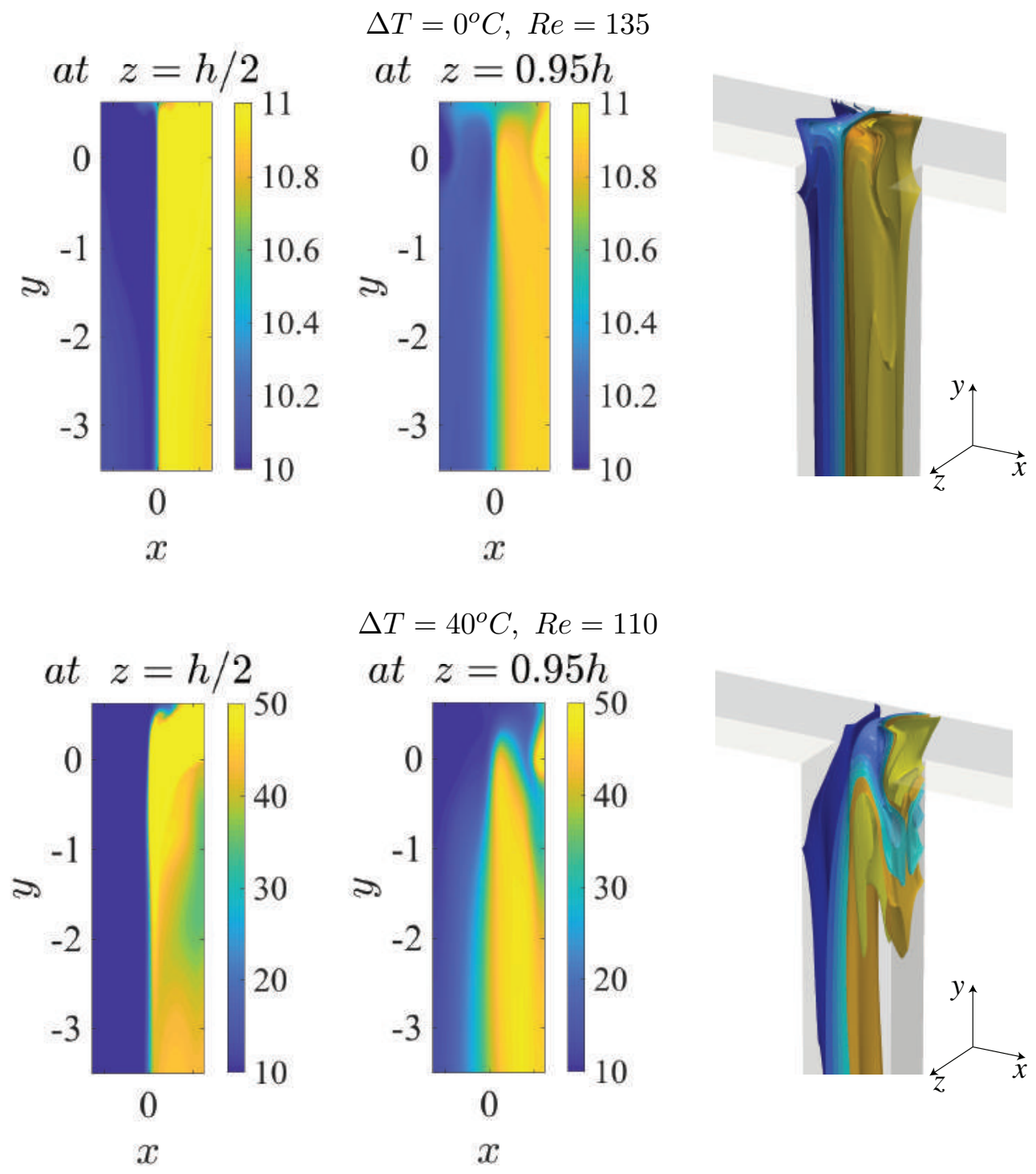

$$
\Delta T=40^{\circ} C, R e=110
$$
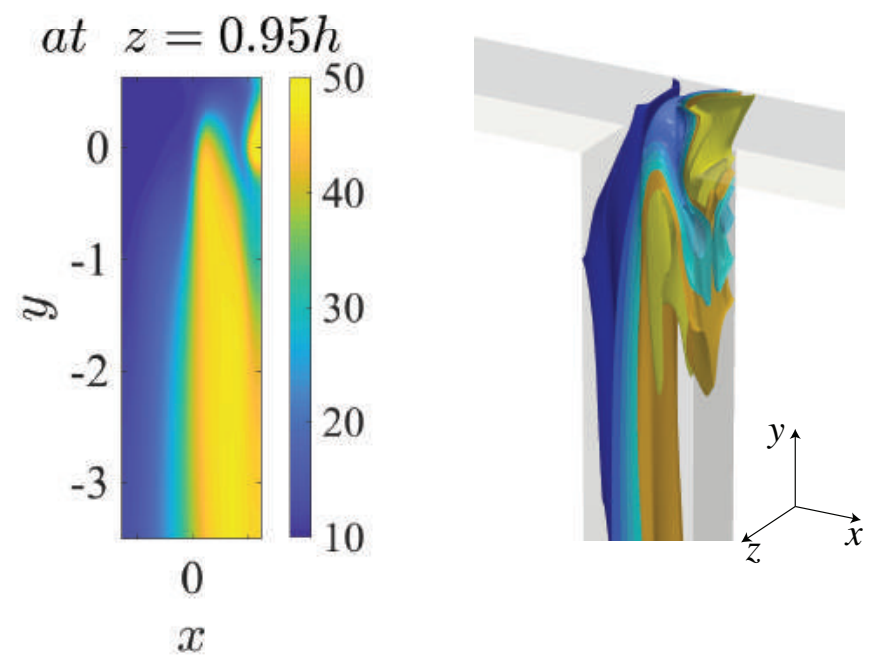

Figure 8. Distribution of temperature in the vortex regime for different values of the temperature jump $\Delta T=T_{2}-T_{1}$ at two selected sections in $x y$-plane, located at $z=h / 2$ and at $z=0.95 h$ different $\Delta T$ are reported at the same sections of figure ?? and figure ??.

\section{B. Global stability analysis}

Stability analysis is carried out here to further investigate the flow instability leading from the vortex to the engulfment regime and how it is possibly affected by $\Delta T$ and by the consequent variation of viscosity. As a first step, the critical Reynolds number for this instability $R e_{c r}$, already estimated by DNS in Section ??, can be also evaluated by 

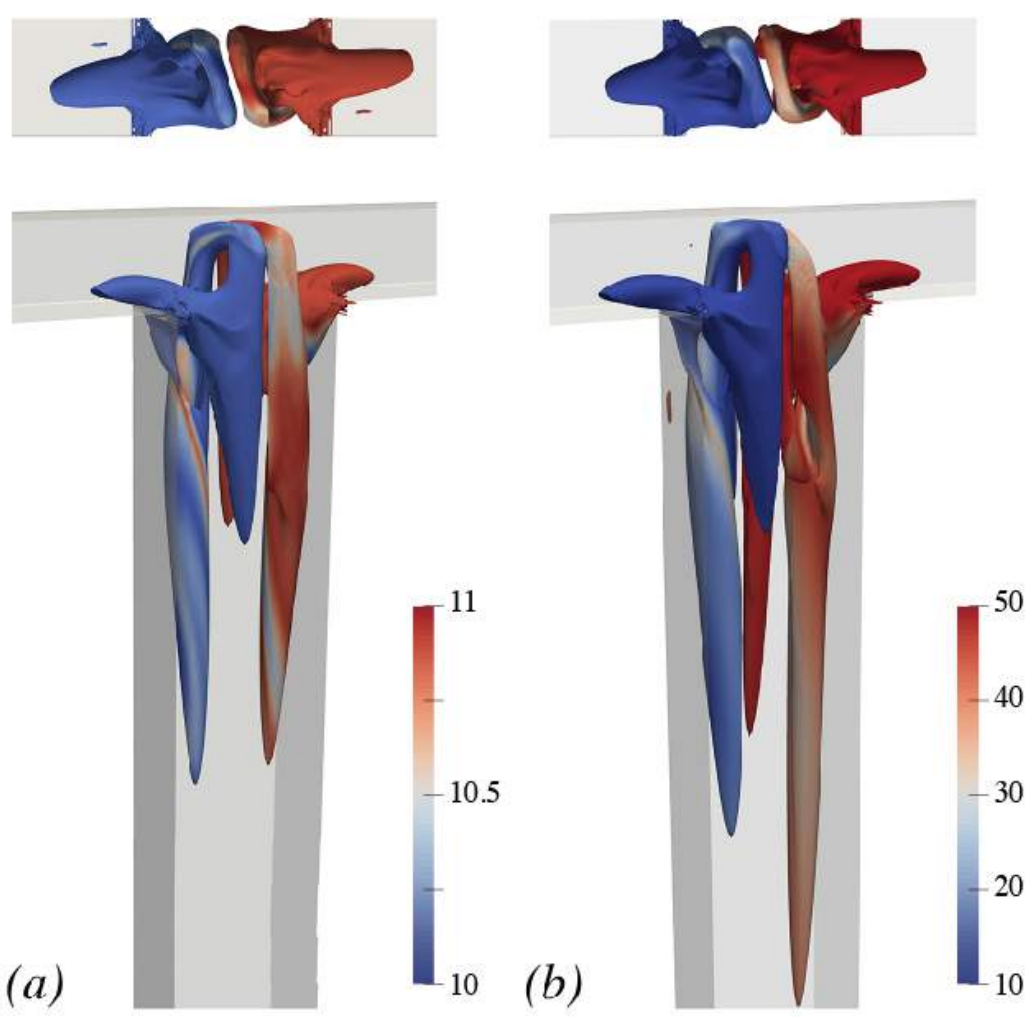

Figure 9. Top and lateral views of the $3 \mathrm{D}$ vortical structures identified by the $\lambda 2$ criterion ([? ]) for the vortex regime at (a) $\Delta T=0^{\circ} \mathrm{C}$ and $R e=160$ and (b) $\Delta T=40^{\circ} \mathrm{C}$ and $R e=120$; the color on the isosurfaces indicate the value of temperature (or of the passive scalar in the case $\Delta T=0^{\circ} \mathrm{C}$ )

stability analysis. This has been done by considering, for each value of $\Delta T$, several values of subcritical flow Reynolds numbers $R e_{j}$. For each value of $R e_{j}$ the stability analysis is applied, identifying the least stable mode and estimating its growth rate $\lambda_{j}=\lambda\left(R e_{j}\right)$. The data so collected are then used to extrapolate the behaviour of the growth rate as a function of the Re, i.e. $\lambda(R e)$, and to approximate the $R e_{c r}$ as the value of $R e$ that corresponds to a null growth rate, i.e. $\lambda\left(R e=R e_{c r}\right) \simeq 0$. We remind that the stability analysis has been carried out here by dedicated numerical tools, described in section ??, which are completely different from the numerical method used for DNS. For this reason slight discrepancies between the two different estimations of $R e_{c r}$ have been found. In particular, the maximum difference has been obtained for $\Delta T=40^{\circ} \mathrm{C}$, where DNS predicts $110 \leq R e_{c r} \leq 120$ while according to the stability analysis we should have $R e_{c r} \simeq 105$. The reasons for this discrepancy, even if rather small, are not fully clear to the authors. As previously said, they might be ascribed to the different methods and spatial resolutions employed. Another possible cause of discrepancy 
$\Delta T=0^{\circ} C, R e=160$
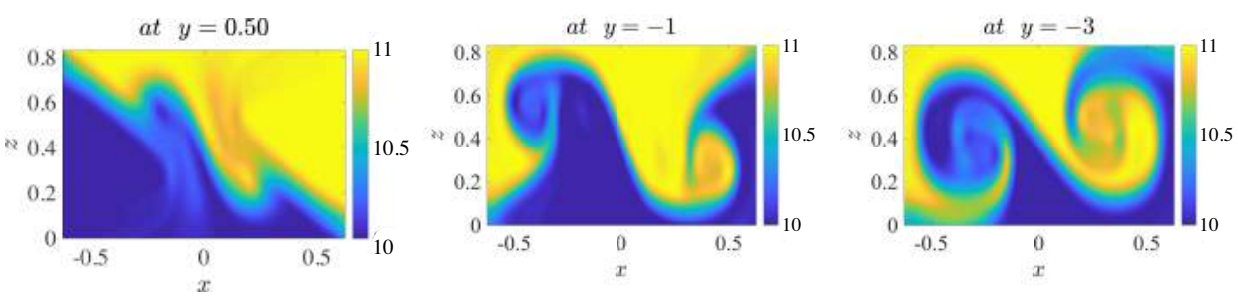

$\Delta T=10^{\circ} C, R e=130$
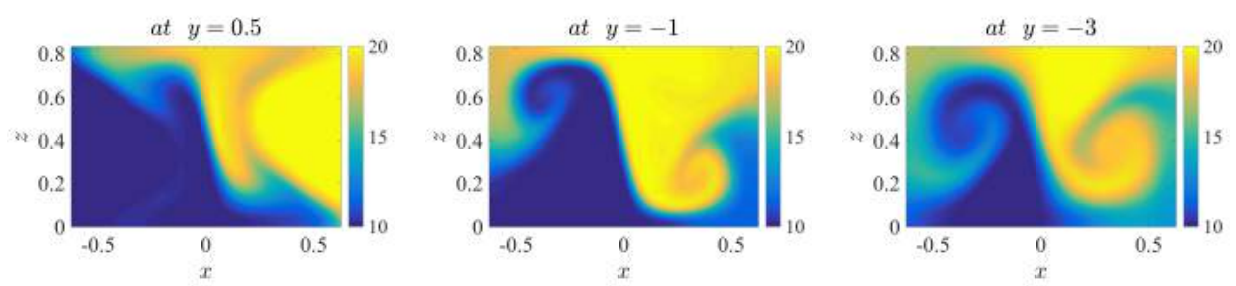

$\Delta T=20^{\circ} C, \quad R e=125$
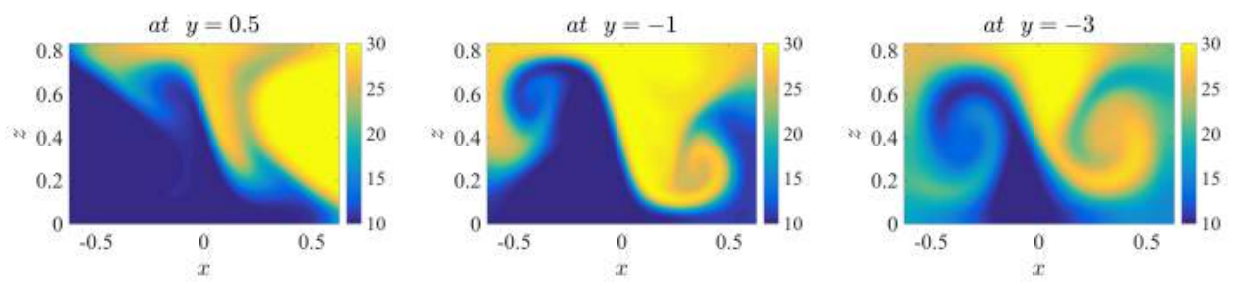

$$
\Delta T=30^{\circ} C, R e=120
$$
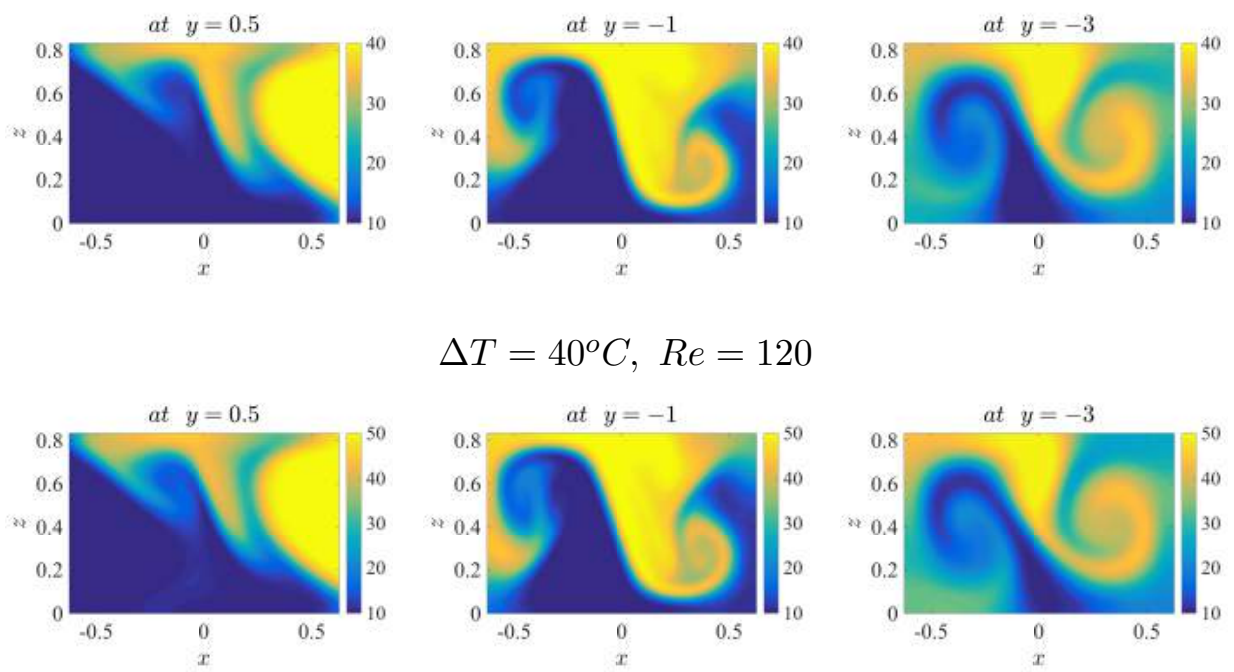

$$
\Delta T=40^{\circ} C, R e=120
$$
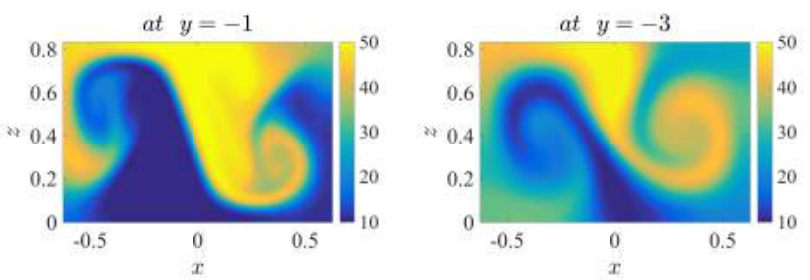

Figure 10. Distribution of temperature in the engulfment regime for different values of the temperature jump $\Delta T=T_{2}-T_{1}$ 


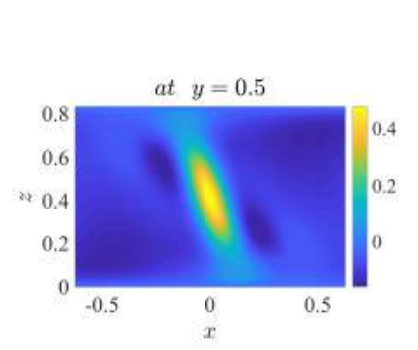

$\Delta T=0^{\circ} C, R e=160$
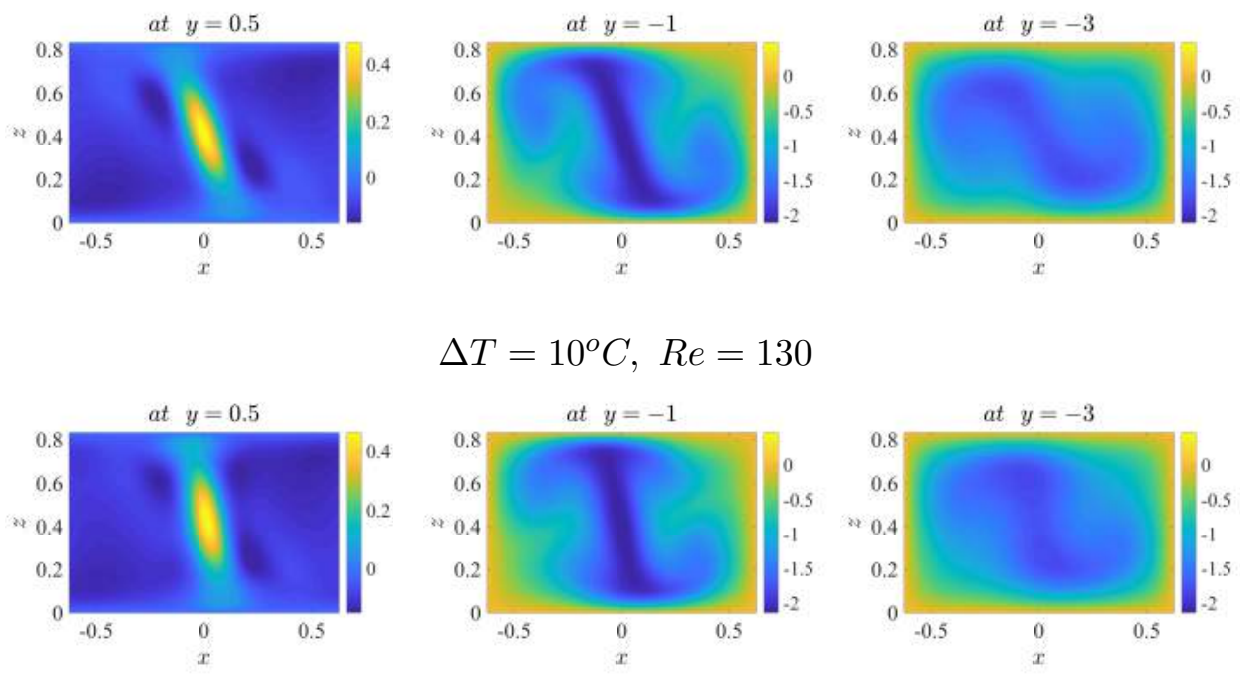

$\Delta T=10^{\circ} C, \quad R e=130$
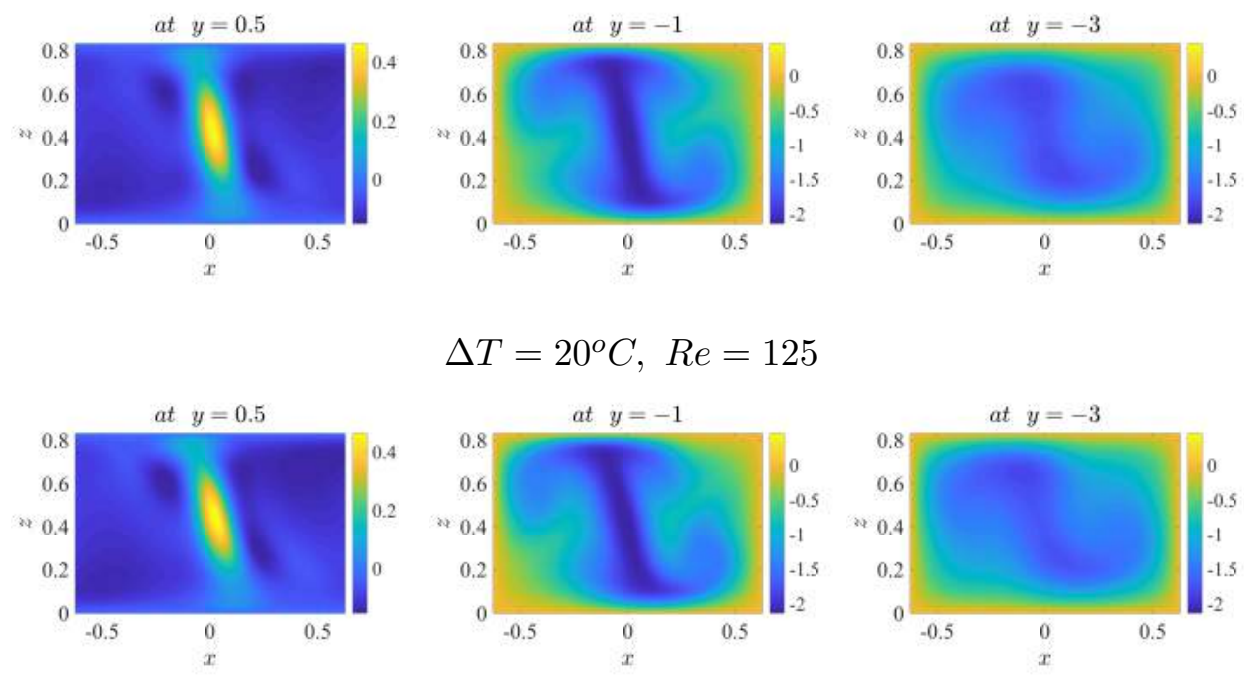

$\Delta T=20^{\circ} C, R e=125$
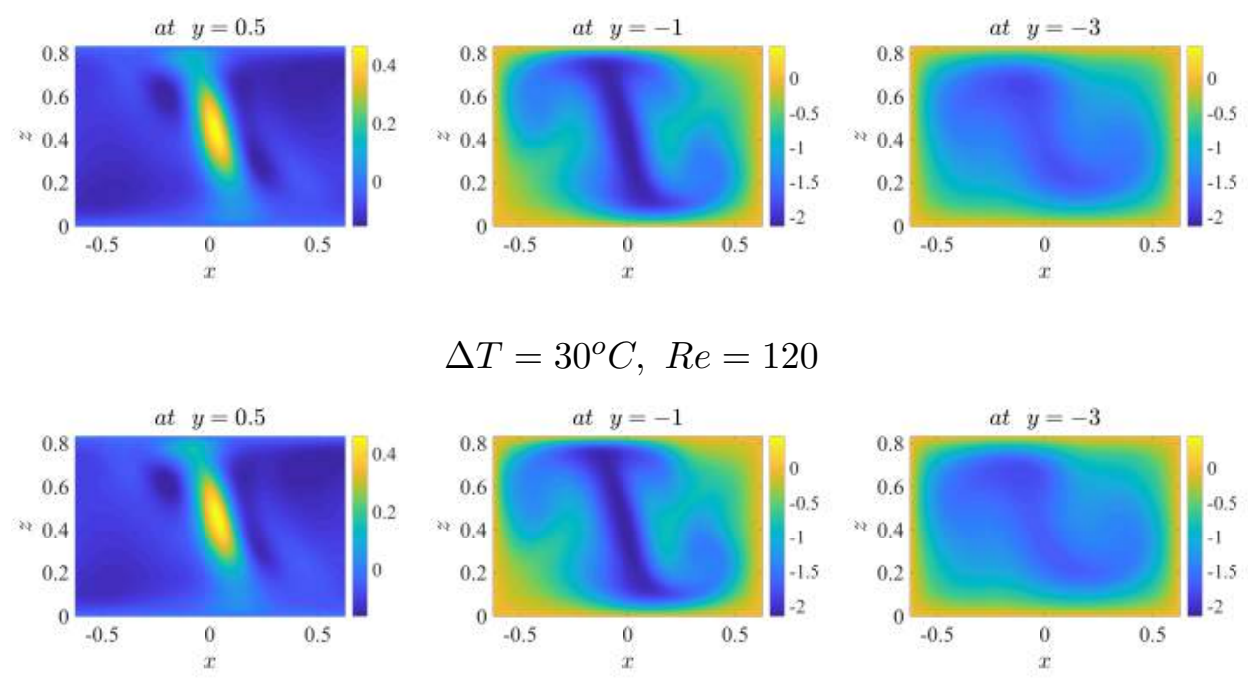

$\Delta T=30^{\circ} C, \quad R e=120$
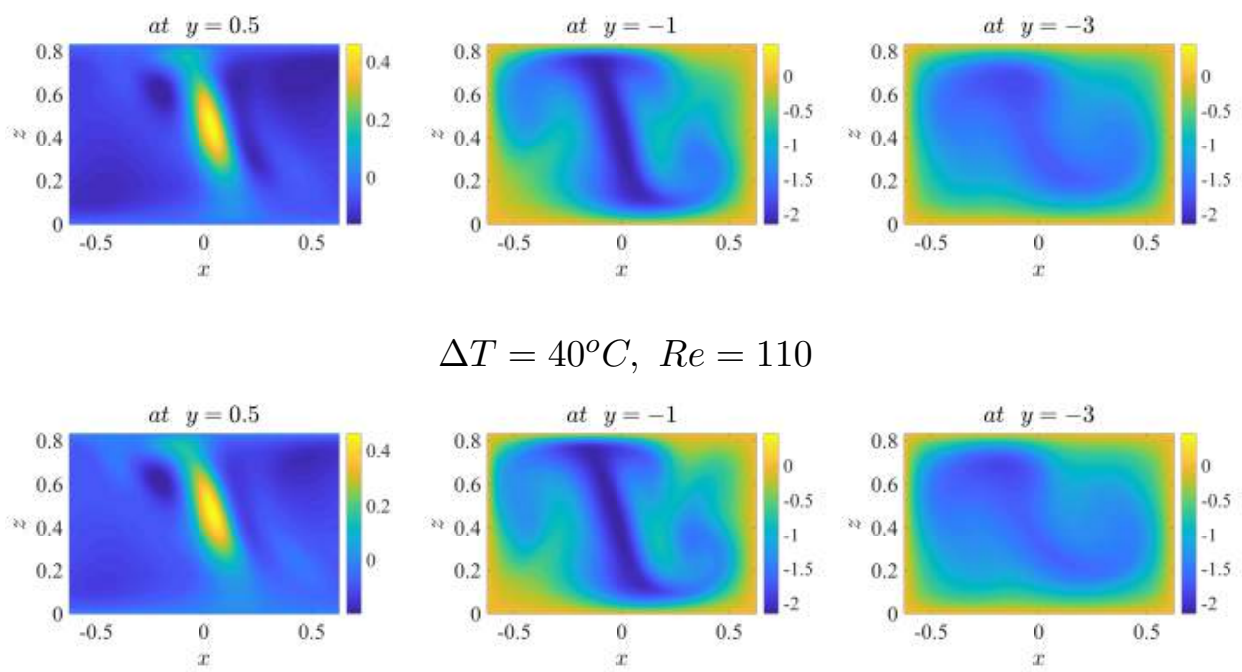

$$
\Delta T=40^{\circ} C, \quad R e=110
$$
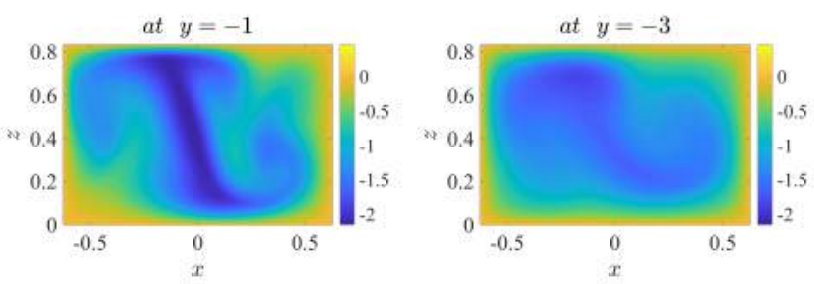

Figure 11. Distribution of the velocity in $y$ direction in the engulfment regime for different values of the temperature jump $\Delta T=T_{2}-T_{1}$ at three selected sections in $x z$-plane at $y=0.5,-1,-3$ 

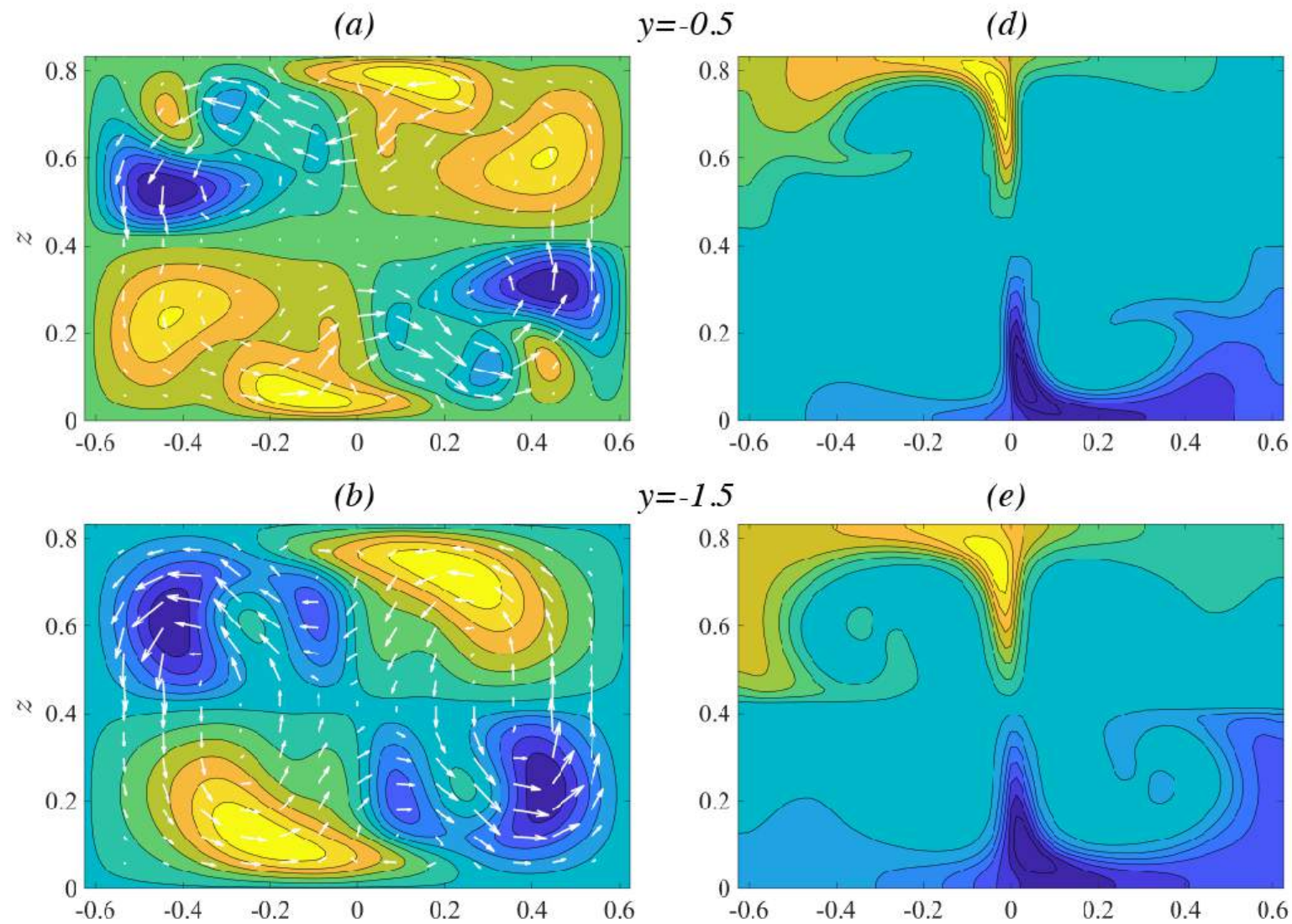

$y=-1.5$

(e)

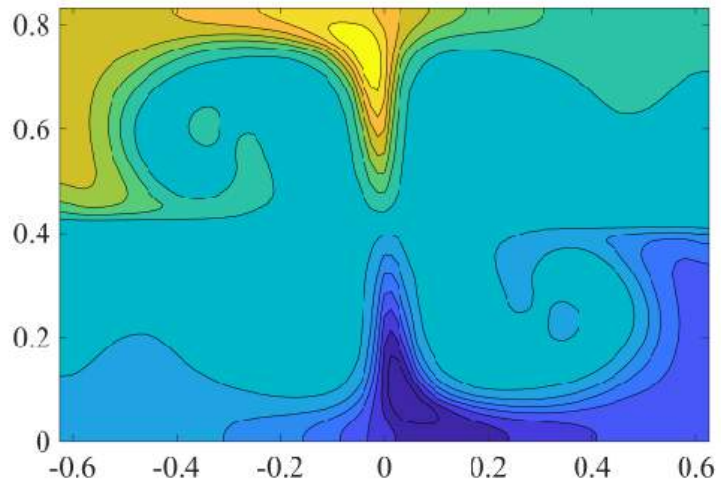

(c) $y=-3$

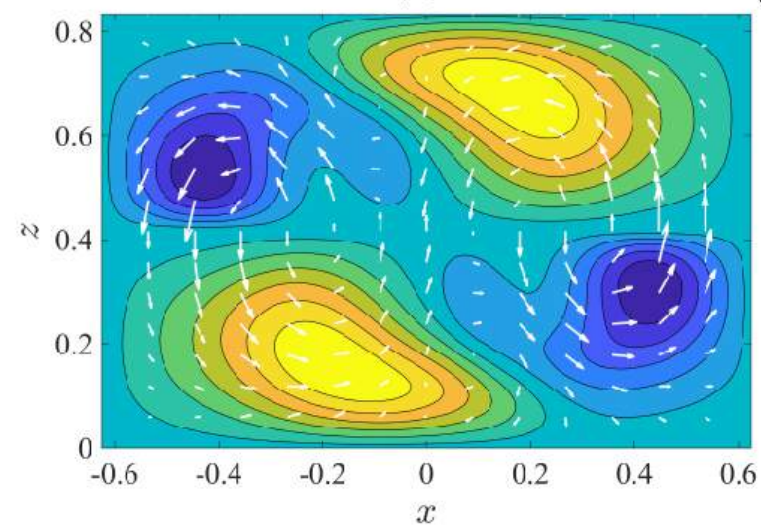

(f)

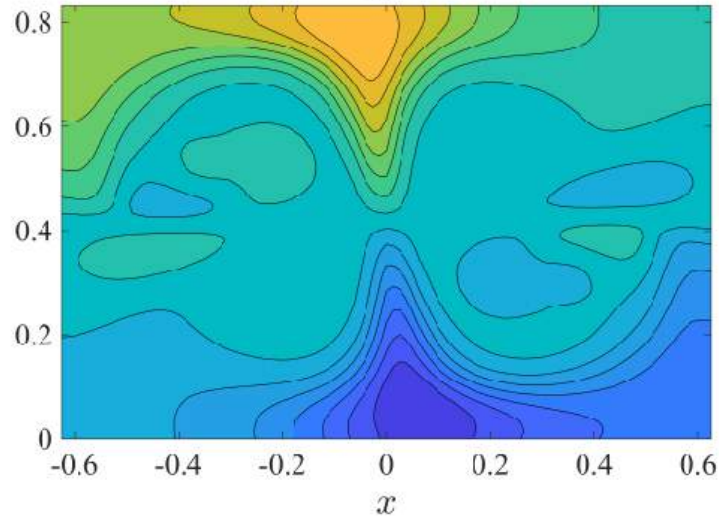

Figure 12. Leading direct global mode for the case $\Delta T=10^{\circ} \mathrm{C}$ and $R e=120$ at three different sections: (a-d) $y=-0.5$, (b-e) $y=-1.5$ and (c-f) $y=-3$. Left column: the color contours represent the y-component of the velocity field and the vectors the in-plane velocity; right column: temperature perturbation field.

lies in the fact that in DNS the simulation times to identify the engulfment instability may be very large when growth factors are low, as it may happen near the critical conditions. 

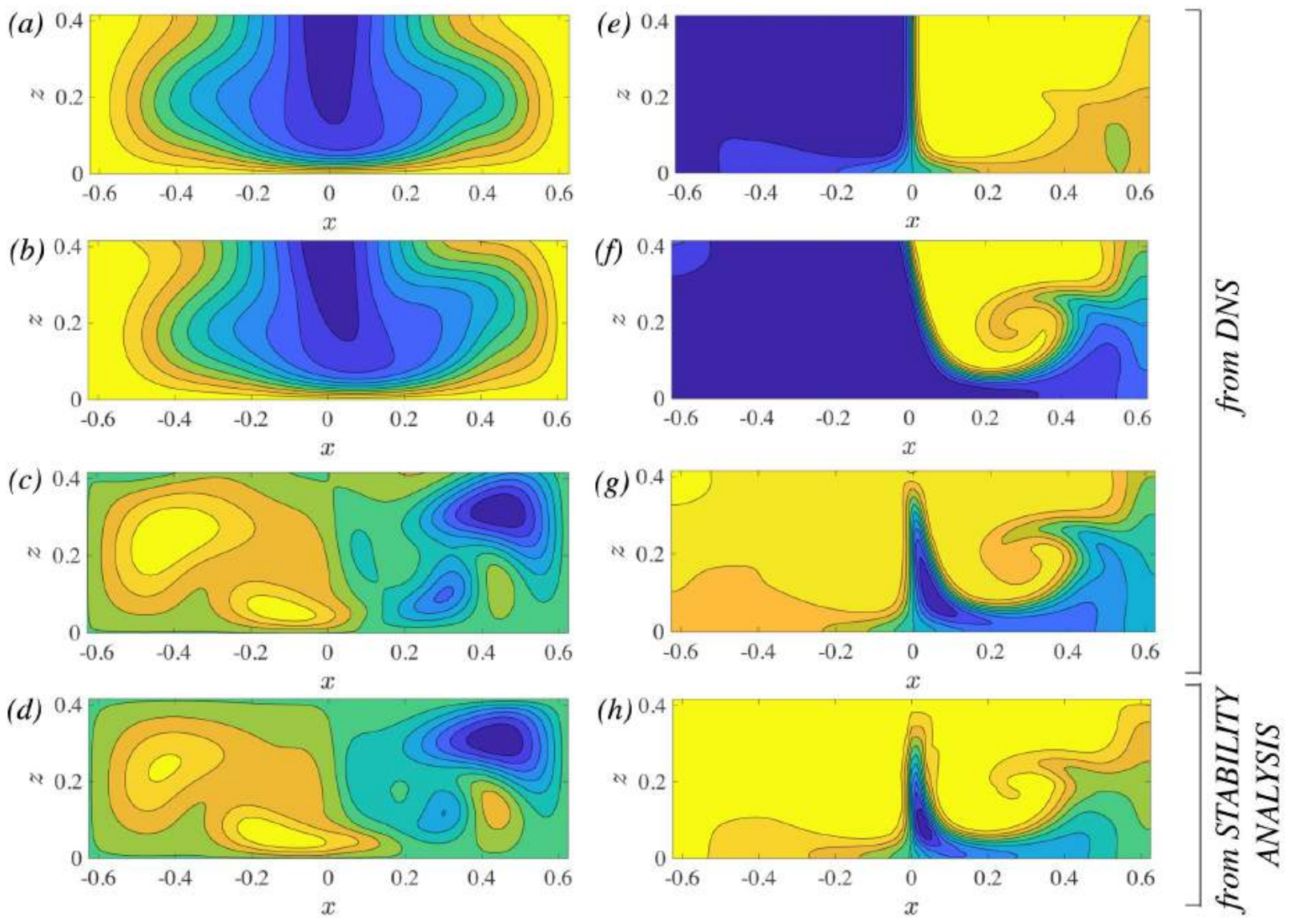

Figure 13. DNS Velocity ( $y$ component) and temperature fields at $y=-0.5$ before (a)-(e) and after (b)-(f) the engulfment instability for $\Delta T=10^{\circ} \mathrm{C}$; the velocity and temperature fields in subfigures (c)-(g) are the difference between the fields (b)-(f) and those in (a)-(e), i.e. the difference fields between the post-enguflment and the pre-engulment ones; velocity ( $y$ component) and temperature eigenmodes obtained by stability analysis are depicted in subfigures (d) and (h) and normalised so as to ease comparison with (c) and (g).

Thus, there might be the possibility that a point which is identified as stable in the vortex regime is slightly unstable but this eventuality has not been shown by DNS.

However, despite the mild differences in terms of $R e_{c r}$, it can be shown that there is a close agreement between the least stable mode identified by global stability analysis (an example of the shape of the leading global mode is shown in figure ??) and the results obtained by DNS, confirming that the stability analysis correctly identifies the mode which is responsible for the onset of the engulfment regime. This can be demonstrated by comparing the spatial shape of the eigenmode identified by stability analysis with the flow field $\left(\Delta \mathbf{v}_{*}, \Delta p_{*}, \Delta T_{*}\right)$ 
(a)

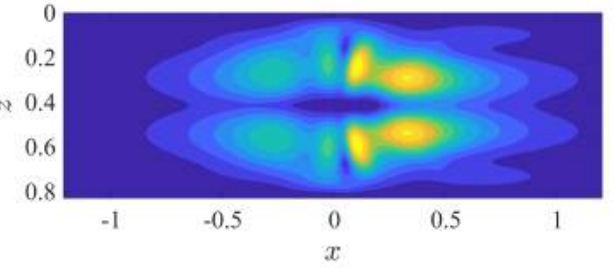

(b)

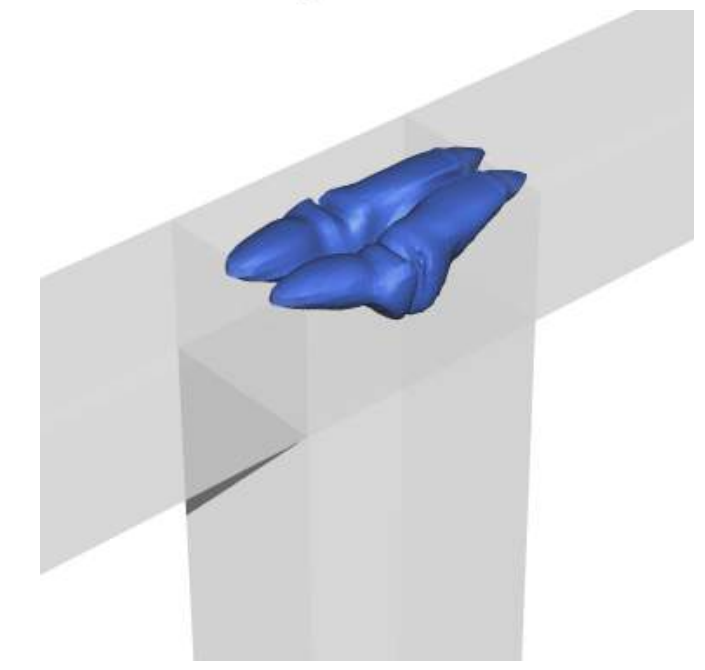

(c)

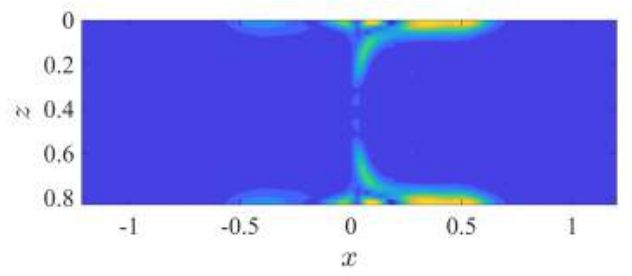

(d)

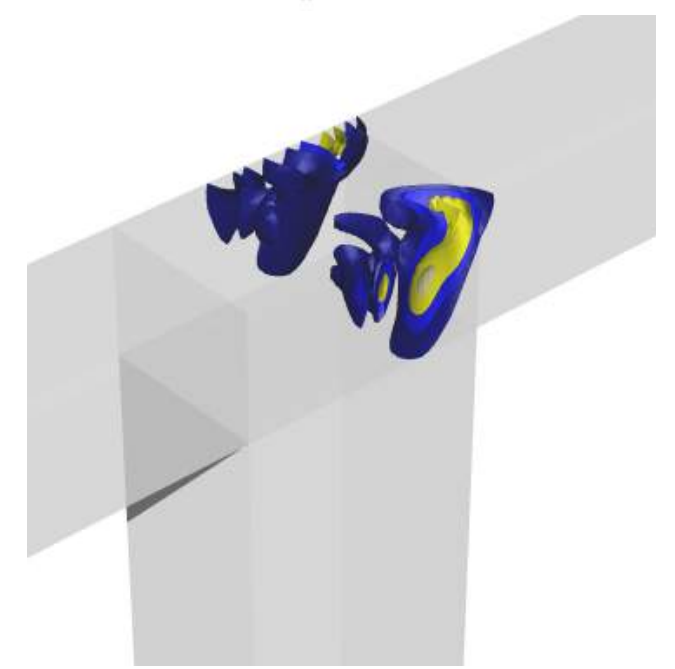

Figure 14. Representative sections (top) and iso-surfaces (bottom) of the sensitivity maps (a) $S_{M}\left(\mathbf{x}_{*}\right)$ and $(\mathrm{b}) S_{T}\left(\mathbf{x}_{*}\right)\left(\Delta T=10^{\circ} \mathrm{C}\right.$ and $\left.R e=120\right)$

obtained by subtracting the flow field obtained in DNS at a Reynolds number slightly lower than $R e_{c r}$ (vortical regime, see for instance figures ??(a) and (e)) to the one obtained at Re slightly larger than $R e_{c r}$ (engulfment regime, see for instance figures ??(b) and (f)). The resulting velocity and temperature fields $\left(\Delta \mathbf{v}_{*}, \Delta p_{*}, \Delta T_{*}\right)$ are reported at the section $y=-0.5$ for $\Delta T=10^{\circ} \mathrm{C}$ in figures ??(c) and $(\mathrm{g})$, respectively. Figure ?? allows the comparison between $\left(\Delta \mathbf{v}_{*}, \Delta p_{*}, \Delta T_{*}\right)$ and the stability eigenmode, which is reported for the same case in figures ??(d) (y-velocity) and (h) (temperature). As can be evinced by the figure, the difference field $\left(\Delta \mathbf{v}_{*}, \Delta p_{*}, \Delta T_{*}\right)$ obtained by DNS, which is representative of the way in which the engulfment instability grows in slightly supercritical conditions over the vortex regime, is in excellent agreement with the least stable eigenmode identified by the stability analysis.

Once demonstrated that the stability analysis allows the identification of the eigenmode leading to the engulfment regime, it is possible to apply the sensitivity analysis described in section ?? so as to locate the core of the instability. We remind that, following [? ], the core of the instability is the region of the flow where the instability takes origin and where it is 
most sensitive to a possible control. The instability core is identified as the region where the sensitivity maps $S_{M}\left(\mathbf{x}_{*}\right)$ and $S_{T}\left(\mathbf{x}_{*}\right)$ (see equation (??)) take values significantly different from 0 . The isosurfaces delimiting these regions are reported in figure ?? for the case at $\Delta T=10^{\circ} \mathrm{C}$ and $R e=120$ (slight subcritical conditions), showing that the core regions are well delimited in space and confined at the confluence region of the two inlet channels. The region where $S_{T}\left(\mathbf{x}_{*}\right)$ is non-null, i.e. where the instability is sensitive to a structural perturbation of the temperature equation, is almost included into the region where also $S_{M}\left(\mathbf{x}_{*}\right)$ is non-null. Moreover, the global core of the instability, which is dominated by the sensitivity map $S_{M}\left(\mathbf{x}_{*}\right)$, is very similar to that already found for the case of uniform fluid properties $(\Delta T=0)$ (see $[?]$ for detailed comments). The only difference is the slight asymmetry of the core with respect to the plane $x=0$, which is related to the asymmetry of the baseflow already discussed in the previous section. From this observation we can argue that the onset of the engulfment regime is mainly related to a mechanism which is dominated by the momentum equation and by the velocity field, while the effect of the temperature field is to modify the scenario from a quantitative viewpoint but not qualitatively, i.e. without altering the physical origin of the instability. To further support this speculation, different global stability analyses have been carried out on artificially modified system of equations. In particular, by inspecting the stability equations (??), it is possible to identify 2 terms which couple the momentum and the temperature linearised equations: the third term $\left(\hat{\mathbf{v}}_{*} \cdot \nabla_{*} T_{b *}\right)$ on the LHS of Eq. (??c), labelled in the following as T1, and the last term of the RHS in Eq. (??b), denoted here as M1. The term T1 is a source term for $\hat{T}_{*}$ and represents the temperature fluctuations caused by the eigenmode velocity $\hat{\mathbf{v}}_{*}$ moving the fluid particles in the base temperature field $T_{b *}$. The term M1 takes into account the variation of the fluid viscosity in the momentum equation caused by the temperature fluctuations $\hat{T}_{*}$. The role of the temperature equation in the engulfment instability can be then highlighted performing the following tests:

1. Test\#1 - The term T1 is neglected. This implies that the momentum equation depends on the temperature fluctuations $\hat{T}_{*}$, while the temperature equation is decoupled from the momentum equation and depends only on the baseflow velocity $\mathbf{V}_{\mathbf{b} *}$.

2. Test\#2 - The term M1 is neglected, meaning that the temperature equation depends on $\hat{\mathbf{v}}_{*}$, while the momentum equation is decoupled from the temperature equation and 

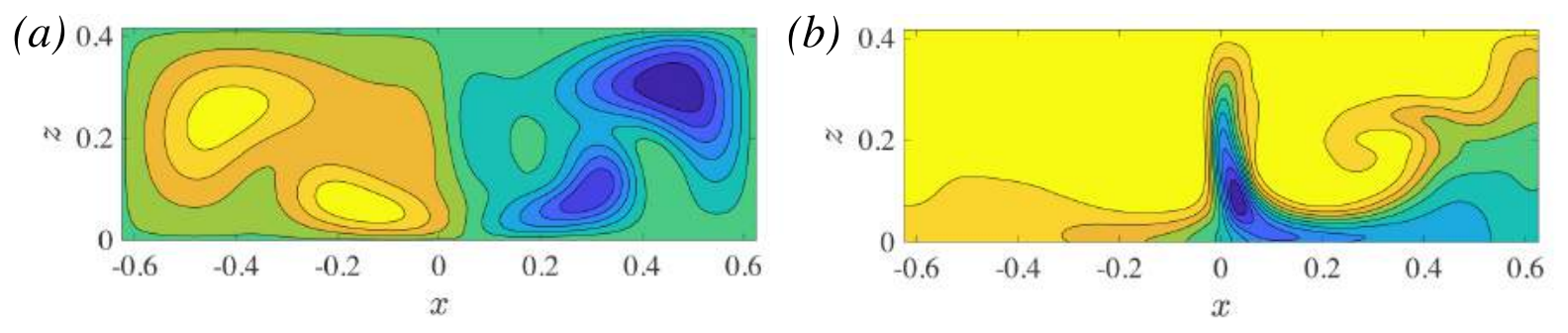

Figure 15. Eigenmodes related to the engulfment instability at $\Delta T=10^{\circ} \mathrm{C}$ and $R e=120$ resulting from the 2 tests described in the text: (a) velocity field in the $y$ direction obtained in both tests 1 and 2 (fields are identical so only one is reported) and (b) temperature field obtained in test 2.

depends only on the baseflow temperature $T_{b *}$ but not on the temperature fluctuations.

Focusing on the case at $\Delta T=10^{\circ} \mathrm{C}$ and $R e=120$, the leading eigenvalue identified in Test\#1 is very close to the one obtained with the full set of equations. A good comparison has been also found between the shape of the velocity field of the corresponding eigenmode, reported in figure ??(a), and the true eigenmode, previously shown in figure ??(d). However, the associated temperature eigenmode, which depends now on a convection-diffusion equation decoupled from the momentum equation, is null to machine accuracy. This result demonstrates that the source for temperature fluctuations in the engulfment instability is driven by the velocity eigenmode through the term $\mathrm{T} 1$ and temperature equation does not drive any new instability mechanism.

On the other hand, the least stable eigenvalue predicted by the Test\#2 is also similar to that of the full stability analysis but, at difference with Test\#1, in this second case the corresponding temperature perturbation field is not null. In particular, the temperature eigenmode, reported in figure ??(b), is now very similar to the one obtained when all the terms of the stability equations are retained, as can be evinced by comparison with figure ??(h). This second test further proves that the temperature eigenmode is driven by the velocity field through the term T1.

With the tests described above we have thus demonstrated that the engulfment instability is dictated by the same physical mechanisms highlighted in the case without temperature difference, i.e. with constant viscosity, which have been largely discussed in the dedicated paper [?]. The role of $\Delta T$ is to change quantitatively the stability characteristics via a variation of the viscosity coefficient, but the temperature equation does not add any new 
instability mechanism to the flow.

\section{CONCLUSIONS}

The flow in a T-mixer is investigated when fluids at different temperatures enter the mixer. Water is selected as working fluid at varying temperature, so as to highlight the effect of a significant viscosity variation on the onset of the fluid flow regimes while density variations are negligible. The temperature of water entering from one channel is hold at a constant value equal to $T_{1}=10^{\circ} \mathrm{C}$, while in the other inlet channel temperature is varied in the range $10^{\circ} \mathrm{C} \leq T_{2} \leq 50^{\circ} \mathrm{C}$. This study focus in particular on the transition from the vortex to the engulfment regime in the mixer, engulfment being of engineering interest due to the related enhancement of the mixing between the inlet fluids.

The results of the numerical simulations show that the onset of the engulfment regime is promoted by increasing the temperature jump between the two entering fluids. In particular, the corresponding critical Reynolds numbers $\left(R e_{c r}\right)$ for the onset of the flow bifurcation leading to the engulfment tend to decrease with increasing the temperature difference up to $T_{2}-T_{1}=\Delta T=30^{\circ} \mathrm{C}$. Above this temperature difference, $R e_{c r}$ reaches an almost constant value approximately equal to $R e_{c r}=115$. This behaviour is also confirmed by the evaluation of the degree of mixing between the two entering fluids for the temperature jumps considered here. This second test also shows that the saturation of the mixing in the engulfment regime with increasing $R e$ is faster for larger $\Delta T$. DNS simulations also show that, when the inlet streams have different temperature and hence viscosity, a loss of symmetry is present in the flow filed also in the vortical regime and this promotes the occurrence of the engulfment regime, in which the flow symmetries are definitely broken.

Lastly, three-dimensional global stability analysis has been carried out to further characterise the process leading to the engulfment regime. For each value of $\Delta T$ considered in the selected range specified above, a supercritical pitchfork bifurcation has been identified from the analysis of the eigenvalue spectrum obtained at different values of the flow Reynolds number, following the same procedure proposed by [? ] for the study of a similar flow configuration when $\Delta T=0$. Despite slight discrepancies between the values of $R e_{c r}$ estimated by DNS and by stability analysis, which are however compatible with the different numerical methods used in the two cases, it is shown that the global mode identified by stability ana- 
lysis is the one responsable for the onset of the engulfment regime. Consequently, sensitivity analysis has been applied to localise the core of the instability and to further investigate the role played by the temperature field in the onset of the engulfment. It is shown that the instability core is similar to that already identified for a uniform fluid in [? ]. Indeed, the new part resulting from the temperature equation leads to a high sensitivity region which is almost completely included into that related to the momentum equation, so that the overall sensitivity region is only slightly different.

In order to further investigate the role of the temperature equation on the onset of the identified bifurcation, two artificially modified stability problems have been formulated so as to isolate the role of each one among the two terms of the equations which couple the momentum and the temperature equations. The results obtained and their comparison with the true case demonstrate that the temperature equation does not lead to any new instability mechanism, but engulfment is dictated by the same physical mechanisms highlighted in the case without temperature difference. The effect of the temperature is only to change quantitatively the stability characteristics via a variation of the viscosity coefficient.

\section{ACKNOWLEDGMENTS}

This work was supported by the University of Pisa through the "Progetti di Ricerca di Ateneo PRA 2017-2018" funding program. The authors wish also to thank CINECA computing center (Bologna, Italy) for allowance of computational resources under ISCRA program (class B project "MIMIC").

\section{Appendix A: Derivation of the adjoint stability equations}

We derive here the adjoint stability equations (??) using a classical variational approach. The starting point are the direct stability equations (??), which are concisely indicated as follows:

$$
\mathcal{L} \hat{\mathbf{q}}_{*}=\mathbf{0}
$$

where the vector $\hat{\mathbf{q}}_{*}$ collects the direct eigenmode, i.e. $\hat{\mathbf{q}}_{*}=\left(\hat{\mathbf{v}}_{*}, \hat{p}_{*}, \hat{T}_{*}\right)^{T}$ Equation (??) is introduced through associated Lagrange multipliers $\tilde{\mathbf{q}}_{*}=\left(\tilde{\mathbf{v}}_{*},-\tilde{p}_{*}, \tilde{T}_{*}\right)^{T}$ in an augmented 
functional $J$ :

$$
J=\sigma-\left\langle\tilde{\mathbf{q}}_{*}, \mathcal{L} \hat{\mathbf{q}}_{*}\right\rangle
$$

Imposing stationarity of $J$ with respect to generic variation of the adjoint variables, $\delta \tilde{\mathbf{q}}_{*}$, i.e. $\delta J=-\left\langle\delta \tilde{\mathbf{q}}_{*}, \mathcal{L} \hat{\mathbf{q}}_{*}\right\rangle=0$, leads to equation (??), which is the starting direct problem included here as a constraint. For the derivation of the adjoint problems it is necessary to find the adjoint operator $\mathcal{L}^{+}$, which is defined as follows:

$$
\left\langle\tilde{\mathbf{q}}_{*}, \mathcal{L} \hat{\mathbf{q}}_{*}\right\rangle=\left\langle\mathcal{L}^{+} \tilde{\mathbf{q}}_{*}, \hat{\mathbf{q}}_{*}\right\rangle
$$

Following the definition in equation (??), the operator $\mathcal{L}^{+}$and the associated boundary conditions are found using integration by parts so as to shift the application differential operators from $\hat{\mathbf{q}}_{*}$ to $\tilde{\mathbf{q}}_{*}$. The boundary integrals resulting from this procedure needs to be nullified by properly selected boundary conditions to be applied to the adjoint variables $\tilde{\mathbf{q}}_{*}$. Once $\mathcal{L}^{+}$has been found, the augmented functional can be written as:

$$
J=\sigma-\left\langle\mathcal{L}^{+} \tilde{\mathbf{q}}_{*}, \hat{\mathbf{q}}_{*}\right\rangle
$$

Imposing stationarity of $J$ with respect to generic variation of the direct variables, $\delta \hat{\mathbf{q}}_{*}$, i.e. $\delta J=-\left\langle\mathcal{L}^{+} \tilde{\mathbf{q}}_{*}, \delta \hat{\mathbf{q}}_{*}\right\rangle=0$, leads to the adjoint eigenvalue problem:

$$
\mathcal{L}^{+} \tilde{\mathbf{q}}_{*}=\mathbf{0}
$$

which is supplemented by the natural boundary conditions for $\tilde{\mathbf{q}}_{*}$ which nullify the boundary intergrals arising in the derivation of the operator $\mathcal{L}^{+}$. Once the direct and the adjoint problems are solved, it is possible to find the variation $\delta \sigma$ of the selected eigenvalue for a generic variation of the linearised operator, $\delta \mathcal{L}$, as follow:

$$
\delta \sigma=-\left\langle\tilde{\mathbf{q}}_{*}, \delta \mathcal{L} \hat{\mathbf{q}}_{*}\right\rangle
$$

and this is done in Section ?? for investigating the sensitivity of the instability to a particular structural perturbation of the linearised Navier Stokes equations.

In the following we will concisely detail the passage formally given in equation (??). Integration by parts is applied to the term $\left\langle\tilde{\mathbf{q}}_{*}, \mathcal{L} \hat{\mathbf{q}}_{*}\right\rangle$ until all differential operators are moved from $\hat{\mathbf{q}}_{*}$ to $\tilde{\mathbf{q}}_{*}$, which implies one integration by parts for first-order terms and two for second-order 
terms, and the final result is the following:

$$
\begin{aligned}
& \left\langle\tilde{\mathbf{q}}_{*}, \mathcal{L} \hat{\mathbf{q}}_{*}\right\rangle=-\left\langle\tilde{p}_{*}, \nabla_{*} \cdot \hat{\mathbf{v}}_{*}\right\rangle+ \\
& \left\langle\tilde{\mathbf{V}}_{*}, \sigma \hat{\mathbf{V}}_{*}+\mathbf{V}_{\mathbf{b} *} \cdot \nabla_{*} \hat{\mathbf{V}}_{*}+\hat{\mathbf{v}}_{*} \cdot \nabla_{*} \mathbf{V}_{\mathbf{b} *}+\nabla_{*} \hat{p}_{*}-\nabla_{*} \cdot\left[\alpha\left(T_{b *}\right)\left(\nabla_{*} \hat{\mathbf{V}}_{*}+\nabla_{*}^{T} \hat{\mathbf{V}}_{*}\right)\right]+\right. \\
& \left.-\nabla_{*} \cdot\left[\left.\frac{\partial \alpha(y)}{\partial y}\right|_{y=T_{b *}}\left(\nabla_{*} \mathbf{V}_{\mathbf{b} *}+\nabla_{*}^{T} \mathbf{V}_{\mathbf{b} *}\right)\right] \hat{T}_{*}\right\rangle+ \\
& \left\langle\tilde{T}_{*}, \sigma \hat{T}_{*}+\mathbf{V}_{\mathbf{b} *} \cdot \nabla_{*} \hat{T}_{*}+\hat{\mathbf{v}}_{*} \cdot \nabla_{*} T_{b *}-\nabla_{*} \cdot\left(\beta\left(T_{b *}\right) \nabla_{*} \hat{T}_{*}\right)+\right. \\
& \left.-\nabla_{*} \cdot\left(\left.\frac{\partial \beta(y)}{\partial y}\right|_{y=T_{b *}} \nabla_{*} T_{b *}\right) \hat{T}_{*}\right\rangle= \\
& -\left\langle\nabla_{*} \cdot \tilde{\mathbf{v}}_{*}, \hat{p}_{*}\right\rangle+ \\
& \left\langle\sigma^{*} \tilde{\mathbf{v}}_{*}-\mathbf{V}_{\mathbf{b} *} \cdot \nabla_{*} \tilde{\mathbf{V}}_{*}+\nabla_{*} \mathbf{V}_{\mathbf{b} *} \cdot \tilde{\mathbf{v}}_{*}+\nabla_{*} \tilde{p}_{*}, \hat{\mathbf{v}}_{*}\right\rangle+ \\
& +\left\langle-\nabla_{*} \cdot\left[\alpha\left(T_{b *}\right)\left(\nabla_{*} \tilde{\mathbf{v}}_{*}+\nabla_{*}^{T} \tilde{\mathbf{v}}_{*}\right)\right]+\left(\nabla_{*} T_{b *}\right) \tilde{T}_{*}, \hat{\mathbf{v}}_{*}\right\rangle+ \\
& +\left\langle\sigma^{*} \tilde{T}_{*}-\mathbf{V}_{\mathbf{b} *} \cdot \nabla_{*} \tilde{T}_{*}+\frac{1}{2}\left(\nabla_{*} \tilde{\mathbf{V}}_{*}+\nabla_{*}^{T} \tilde{\mathbf{V}}_{*}\right):\left.\left(\nabla_{*} \mathbf{V}_{\mathbf{b} *}+\nabla_{*}^{T} \mathbf{V}_{\mathbf{b} *}\right) \frac{\partial \alpha(y)}{\partial y}\right|_{y=T_{b *}}, \hat{T}_{*}\right\rangle+ \\
& +\left\langle\left.\frac{\partial \beta(y)}{\partial y}\right|_{y=T_{b *}} \nabla_{*} \tilde{T}_{*} \cdot \nabla_{*} T_{b *}-\nabla_{*} \cdot\left(\beta\left(T_{b *}\right) \tilde{T}_{*}\right), \hat{T}_{*}\right\rangle+ \\
& \oint_{\partial V}-\tilde{p}_{*}\left(\hat{\mathbf{v}}_{*} \cdot \mathbf{n}\right) \mathrm{d} S+\oint_{\partial V}\left(\tilde{\mathbf{v}}_{*} \cdot \hat{\mathbf{v}}_{*}\right) \mathbf{V}_{\mathbf{b} *} \cdot \mathbf{n} \mathrm{d} S+\oint_{\partial V} \hat{p}_{*} \tilde{\mathbf{v}}_{*} \cdot \mathbf{n} \mathrm{d} S+ \\
& -\oint_{\partial V} \tilde{\mathbf{v}}_{*} \cdot\left\{\left[\left.\frac{\partial \alpha(y)}{\partial y}\right|_{y=T_{b *}}\left(\nabla_{*} \mathbf{V}_{\mathbf{b} *}+\nabla_{*}^{T} \mathbf{V}_{\mathbf{b} *}\right) \hat{T}_{*}\right] \cdot \mathbf{n}\right\} \mathrm{d} S+ \\
& -\oint_{\partial V} \tilde{\mathbf{v}}_{*} \cdot\left\{\left[\alpha\left(T_{b *}\right)\left(\nabla_{*} \hat{\mathbf{v}}_{*}+\nabla_{*}^{T} \hat{\mathbf{v}}_{*}\right)\right] \cdot \mathbf{n}\right\} \mathrm{d} S+ \\
& +\oint_{\partial V} \hat{\mathbf{v}}_{*} \cdot\left\{\left[\alpha\left(T_{b *}\right)\left(\nabla_{*} \tilde{\mathbf{v}}_{*}+\nabla_{*}^{T} \tilde{\mathbf{v}}_{*}\right)\right] \cdot \mathbf{n}\right\} \mathrm{d} S+ \\
& +\oint_{\partial V} \hat{T}_{*} \tilde{T}_{*}\left(\mathbf{V}_{\mathbf{b} *} \cdot \mathbf{n}\right) \mathrm{d} S-\oint_{\partial V}\left(\left.\tilde{T}_{*} \frac{\partial \beta(y)}{\partial y}\right|_{y=T_{b *}} \hat{T}_{*}\right) \nabla_{*} T_{b *} \cdot \mathbf{n} \mathrm{d} S+ \\
& -\oint_{\partial V} \tilde{T}_{*} \beta\left(T_{b *}\right)\left(\nabla_{*} \hat{T}_{*} \cdot \mathbf{n}\right) \mathrm{d} S+\oint_{\partial V} \hat{T}_{*} \beta\left(T_{b *}\right)\left(\nabla_{*} \tilde{T}_{*} \cdot \mathbf{n}\right) \mathrm{d} S
\end{aligned}
$$


The boundary integrals, i.e. those terms involving a surface integral on the surface bounding the fluid domain, can be rearranged in the following way:

$$
\begin{aligned}
& \oint_{\partial V} \hat{\mathbf{v}}_{*} \cdot\left\{-\tilde{p}_{*} \mathbf{n}+\tilde{\mathbf{v}}_{*}\left(\mathbf{V}_{\mathbf{b} *} \cdot \mathbf{n}\right)+\left[\alpha\left(T_{b *}\right)\left(\nabla_{*} \tilde{\mathbf{V}}_{*}+\nabla_{*}^{T} \tilde{\mathbf{v}}_{*}\right)\right] \cdot \mathbf{n}\right\} \mathrm{d} S+ \\
& +\oint_{\partial V} \tilde{\mathbf{v}}_{*} \cdot\left\{\hat{p}_{*} \mathbf{n}-\left[\left.\frac{\partial \alpha(y)}{\partial y}\right|_{y=T_{b *}}\left(\nabla_{*} \mathbf{V}_{\mathbf{b} *}+\nabla_{*}^{T} \mathbf{V}_{\mathbf{b} *}\right) \hat{T}_{*}\right] \cdot \mathbf{n}-\left[\alpha\left(T_{b *}\right)\left(\nabla_{*} \hat{\mathbf{v}}_{*}+\nabla_{*}^{T} \hat{\mathbf{v}}_{*}\right)\right] \cdot \mathbf{n}\right\} \mathrm{d} S+ \\
& +\oint_{\partial V} \hat{T}_{*}\left[\tilde{T}_{*}\left(\mathbf{V}_{\mathbf{b} *} \cdot \mathbf{n}\right)+\beta\left(T_{b *}\right)\left(\nabla_{*} \tilde{T}_{*} \cdot \mathbf{n}\right)\right] \mathrm{d} S \\
& -\oint_{\partial V}\left[\left(\left.\tilde{T}_{*} \frac{\partial \beta(y)}{\partial y}\right|_{y=T_{b *}} \hat{T}_{*}\right) \nabla_{*} T_{b *} \cdot \mathbf{n}\right] \mathrm{d} S-\oint_{\partial V}\left[\tilde{T}_{*} \beta\left(T_{b *}\right)\left(\nabla_{*} \hat{T}_{*} \cdot \mathbf{n}\right)\right] \mathrm{d} S
\end{aligned}
$$

We remind that the boundary conditions for the base flow $\left(\mathbf{V}_{\mathbf{b} *}, P_{b *}, T_{b *}\right)$ are the following:

$$
\begin{gathered}
\mathbf{V}_{\mathbf{b} *}=\mathbf{f}(\mathbf{x}), \quad T_{b *}=g(\mathbf{x}), \quad \text { on } \Gamma_{I} \\
\mathbf{V}_{\mathbf{b} *}=\mathbf{0}, \quad \nabla_{*} T_{b *} \cdot \mathbf{n}=0, \quad \text { on } \Gamma_{W} \\
{\left[-P_{b *} I+\nabla_{*} \cdot\left(\alpha\left(T_{b *}\right)\left(\nabla_{*} \mathbf{V}_{\mathbf{b} *}+\nabla_{*}^{T} \mathbf{V}_{\mathbf{b} *}\right)\right)\right] \cdot \mathbf{n}=0, \quad \nabla_{*} T_{b *} \cdot \mathbf{n}=0, \quad \text { on } \Gamma_{O}}
\end{gathered}
$$

Moreover, the boundary conditions for the direct eigenmodes in the stability problem, $\left(\hat{\mathbf{v}}_{*}, \hat{p}_{*}, \hat{T}_{*}\right)$, are the following:

$$
\begin{aligned}
& \hat{\mathbf{v}}_{*}=\mathbf{0}, \quad \hat{T}_{*}=0, \quad \text { on } \Gamma_{I} \\
& \hat{\mathbf{v}}_{*}=\mathbf{0}, \quad \nabla_{*} \hat{T}_{*} \cdot \mathbf{n}=0, \quad \text { on } \Gamma_{W} \\
& {\left[\hat{p}_{*} I-\nabla_{*} \cdot\left(\alpha\left(T_{b *}\right)\left(\nabla_{*} \hat{\mathbf{V}}_{*}+\nabla_{*}^{T} \hat{\mathbf{V}}_{*}\right)\right)-\nabla_{*} \cdot\left(\left.\frac{\partial \alpha(y)}{\partial y}\right|_{y=T_{b *}}\left(\nabla_{*} \mathbf{V}_{\mathbf{b} *}+\nabla_{*}^{T} \mathbf{V}_{\mathbf{b} *}\right)\right) \hat{T}_{*}\right] \cdot \mathbf{n}=} \\
& =0, \quad \nabla_{*} \hat{T}_{*} \cdot \mathbf{n}=0, \quad \text { on } \Gamma_{O}
\end{aligned}
$$

Considering that the boundary conditions for the baseflow and for the direct problem and inspecting the boundary terms in Equation (??) it is possible to deduce the natural boundary conditions for the adjoint flow variables such that all the boundary integrals in Equation (??) vanish. In particular, we have:

$$
\begin{gathered}
\tilde{\mathbf{v}}_{*}=\mathbf{0}, \quad \tilde{T}_{*}=0, \quad \text { on } \Gamma_{I} \\
\tilde{\mathbf{v}}_{*}=\mathbf{0}, \quad \nabla_{*} \tilde{T}_{*} \cdot \mathbf{n}=0, \quad \text { on } \Gamma_{W}
\end{gathered}
$$




$$
\begin{aligned}
& -\tilde{p}_{*} \mathbf{n}+\left(\mathbf{V}_{\mathbf{b} *} \cdot \mathbf{n}\right) \tilde{\mathbf{v}}_{*}+\alpha\left(T_{b *}\right)\left(\nabla_{*} \tilde{\mathbf{v}}_{*}+\nabla_{*}^{T} \tilde{\mathbf{v}}_{*}\right) \cdot \mathbf{n}=\mathbf{0} \quad \text { on } \Gamma_{O} \\
& \left(\mathbf{V}_{\mathbf{b} *} \cdot \mathbf{n}\right) \tilde{T}_{*}+\beta\left(T_{b *}\right) \nabla_{*} \tilde{T}_{*} \cdot \mathbf{n}=0 \quad \text { on } \Gamma_{O}
\end{aligned}
$$

With the above mentioned boundary conditions, all boundary integrals vanish and, inspecting Equation (??) and remembering the definition of the adjoint operator $\mathcal{L}^{+}$given in Equation (??) it is possible to deduce straightforwardly the adjoint Equations (??).

[] Andreussi, T., Galletti, C., Mauri, R., Camarri, S. \& Salvetti, M. V. 2015 Flow regimes in T-shaped micro-mixers. Comput. Chem. Eng. 76 (2015), 150-159.

[] Bothe, D., Stemich, C. \& Warnecke, H.J. 2006 Fluid mixing in a T-shaped micro-mixer. Chemical Engineering Science 61 (9), 2950-2958.

[] Camarri, S. 2015 Flow control design inspired by linear stability analysis. Acta Mechanica 226 (4), 979-1010.

[] Cherlo, S.K. \& Pushpavanam, S. 2010 Effect of depth on onset of engulfment in rectangular micro-channels. Chem. Eng. Sci. 65 (24), 6486-6490.

[] Cortes-Quiroz, C.A., Azarbadegan, A. \& Zangeneh, M. 2017 Effect of channel aspect ratio of 3-d t-mixer on flow patterns and convective mixing for a wide range of reynolds number. Sensors and Actuators, B: Chemical 239, 1153-1176.

[] D. Sipp, O. Marquet, P. Meliga \& Barbagallo, A. 2010 Dynamics and control of global instabilities in open-flows: A linearized approach. Appl. Mech. Rev. 63, 030801.

[] Drazin, P. G. \& Reid, W. H. 1981 Hydrodynamic stability. Cambridge University Press.

[] Dreher, S., Kockmann, N. \& Woias, P. 2009 Characterization of Laminar Transient Flow Regimes and Mixing in T-shaped Micromixers. Heat Transf. Eng. 30 (1-2), 91-100.

[] Elvira, K.S., I Solvas, X.C., Wootton, R.C.R. \& Demello, A.J. 2013 The past, present and potential for microfluidic reactor technology in chemical synthesis. Nature Chemistry 5 (11), 905-915.

[] Engler, M., Kockmann, N., Kiefer, T. \& Woias, P. 2004 Numerical and experimental investigations on liquid mixing in static micromixers. Chem. Eng. J. 101 (1-3), 315-322.

[] ESDU-68023 1978 Thermal conductivity, viscosity, heat capacity, density and Prandtl number of solid, liquid and gaseous heavy water. Engineering Sciences Data Unit, UK.

[] ESDU-78040 1978 Viscosity od water and steam. Engineering Sciences Data Unit, UK. 
[] Fani, A., Camarri, S. \& Salvetti, M.V. 2013 Investigation of the steady engulfment regime in a three-dimensional T-mixer. Phys. Fluids 25 (064102).

[] Fani, A., Camarri, S. \& Salvetti, M.V. 2014 Unsteady asymmetric engulfment regime in a t-mixer. Physics of Fluids (1994-present) 26 (7), 074101.

[] Galletti, C., Arcolini, G., Brunazzi, E. \& Mauri, R. 2015 Mixing of binary fluids with composition-dependent viscosity in a t-shaped micro-device. Chemical Engineering Science 123, $300-310$.

[] Galletti, C., Brunazzi, E. \& Mauri, R. 2017 Unsteady mixing of binary liquid mixtures with composition-dependent viscosity. Chem. Eng. Sci. 164, 333-343.

[] Giannetti, F. \& Luchini, P. 2007 Structural sensitivity of the first instability of the cylinder wake. J. Fluid Mech. 581, 167-197.

[] Hoffmann, M., Schlüter, M. \& RÄBiger, N. 2006 Experimental investigation of liquidliquid mixing in T-shaped micro-mixers using -LIF and -PIV. Chem. Eng. Sci. 61 (9), $2968-2976$.

[] Hnon, P. \& SAAD, Y. 2006 A parallel multistage ilu factorization based on a hierarchical graph decomposition. SIAM Journal on Scientific Computing 28 (6), 2266-2293.

[] Izadpanah, E., Hekmat, M.H., Azimi, H., Hoseini, H. \& Rabiee, M.B. 2018 Numerical simulation of mixing process in t-shaped and dt-shaped micromixers. Chemical Engineering Communications 0 (0), 1-9.

[] Jeong, J. \& Hussain, F. 1995 On the identification of a vortex. J. Fluid Mech. 285, 69-94.

[] Karniadakis, G.E., Israeli, M. \& Orszag, S.A. 1991 High-order splitting methods for the incompressible Navier-Stokes equations. J. Comput. Phys. 97 (2), 414-443.

[] Kockmann, N., Kiefer, T., Engler, M. \& Woias, P. 2006 Convective mixing and chemical reactions in microchannels with high flow rates. Sensors and Actuators, B: Chemical 117 (2), 495-508.

[] Kockmann, N. \& Roberge, D.M. 2011 Transitional flow and related transport phenomena in curved microchannels. Heat Transf. Eng. 32 (7-8), 595-608.

[] Lashgari, I., Tammisola, O., Citro, V., Juniper, M. P. \& Brandt, L. 2014 The planar x-junction flow: stability analysis and control. Journal of Fluid Mechanics 753, 128.

[] Lobasov, A.S. \& Minakov, A.V. 2018 Analyzing mixing quality in a t-shaped micromixer for different fluids properties through numerical simulation. Chemical Engineering and 
Processing: Process Intensification 124, 11-23.

[] Lobasov, A. S., Minakov, A. V. \& Rudyak, V. Ya. 2016 Viscosity effect on the flow patterns in t-type micromixers. Fluid Dynamics 51 (3), 381-388.

[] Marioti, A., Galletti, C., Mauri, R., Salvetti, M.V. \& Brunazzi, E. 2018 Steady and unsteady regimes in a t-shaped micro-mixer: synergic experimental and numerical investigation. Chemical Engineering Journal (in press) .

[] Meliga, P. \& Chomaz, J.-M. 2011 Global modes in a confined impinging jet: application to heat transfer and control. Theor. Comput. Fluid Dyn. 25 (1-4), 179-193.

[] Meliga, P., Sipp, D. \& Chomaz, J.-M. 2010 Effect of compressibility on the global stability of axisymmetric wake flows. J. Fluid Mech. 660, 499-526.

[] Meliga, P., Sipp, D. \& Chomaz, J.-M. 2010 Open-loop control of compressible afterbody flows using adjoint methods. Phys. Fluids 22 (5), 054109.

[] Mettot, C., Sipp, D. \& BÉzard, H. 2014 Quasi-laminar stability and sensitivity analyses for turbulent flows: Prediction of low-frequency unsteadiness and passive control. Phys. Fluids 26 (045112).

[] Minakov, A. V., Rudyak, V. Ya., Gavrilov, A. A. \& Dekterev, A. A. 2012 Mixing in a T-shaped micromixer at moderate Reynolds numbers. Thermophys. Aeromechanics 19 (3), $385-395$.

[] Orsi, G., Roudgar, M., Brunazzi, E., Galletti, C. \& Mauri, R. 2013 Water-ethanol mixing in T-shaped microdevices. Chem. Eng. Sci. 95, 174-183.

[] Poole, R.J., Alfateh, M. \& Gauntlett, A.P. 2013 Bifurcation in a t-channel junction: Effects of aspect ratio and shear-thinning. Chemical Engineering Science 104 (Supplement C), $839-848$.

[] Roberge, D. M., Ducry, L., Bieler, N., Cretton, P. \& Zimmermann, B. 2005 Microreactor technology: A revolution for the fine chemical and pharmaceutical industries ? Chemical Engineering \& Technology 28 (3), 318-323.

[] Rossetti, I. \& Compagnoni, M. 2016 Chemical reaction engineering, process design and scale-up issues at the frontier of synthesis: Flow chemistry. Chemical Engineering Journal 296, $56-70$.

[] Siconolfi, L., Fani, A., Camarri, S. \& Salvetti, M.V. 2016 Effect of geometry modifications on the engulfment in micromixers: Numerical simulations and stability analysis. 
European Journal of Mechanics-B/Fluids 55, 360-366.

[] Soleymani, A., Yousefi, H. \& Turunen, I. 2008 Dimensionless number for identification of flow patterns inside a T-micromixer. Chem. Eng. Sci. 63 (21), 5291-5297.

[] Thomas, S., Ameel, T. \& Guilkey, J. 2010 Mixing kinematics of moderate Reynolds number flows in a T-channel. Phys. Fluids 22 (1), 013601.

[] Yoshida, J.-I., Kim, H. \& Nagaki, A. 2011 Green and sustainable chemical synthesis using flow microreactors. ChemSusChem 4 (3), 331-340.

[] You, J.B., Chor, Y. \& Im, S.G. 2017 Influence of adjusting the inlet channel confluence angle on mixing behaviour in inertial microfluidic mixers. Microfluidics and Nanofluidics $21(7)$. 